\title{
Fitting conics to paracatadioptric projections of lines
}

\author{
João P. Barreto *, Helder Araujo \\ Institute for Systems and Robotics, Department of Electrical and Computer Engineering, University of Coimbra, 3030 Coimbra, Portugal
}

Received 21 May 2004; accepted 13 July 2005

Available online 25 October 2005

\begin{abstract}
The paracatadioptric camera is one of the most popular panoramic systems currently available in the market. It provides a wide field of view by combining a parabolic shaped mirror with a camera inducing an orthographic projection. Previous work proved that the paracatadioptric projection of a line is a conic curve, and that the sensor can be fully calibrated from the image of three or more lines. However, the estimation of the conic curves where the lines are projected is hard to accomplish because of the partial occlusion. In general only a small arc of the conic is visible in the image, and conventional conic fitting techniques are unable to accurately estimate the curve. The present work provides methods to overcome this problem. We show that in uncalibrated paracatadioptric views a set of conic curves is a set of line projections if and only if certain properties are verified. These properties are used to constrain the search space and correctly estimate the curves. The conic fitting is solved naturally by an eigensystem whenever the camera is skewless and the aspect ratio is known. For the general situation the line projections are estimated using non-linear optimization. The set of paracatadioptric lines is used in a geometric construction to determine the camera parameters and calibrate the system. We also propose an algorithm to estimate the conic locus corresponding to a line projection in a calibrated paracatadioptric image. It is proved that the set of all line projections is a hyperplane in the space of conic curves. Since the position of the hyperplane depends only on the sensor parameters, the accuracy of the estimation can be improved by constraining the search to conics lying in this subspace. We show that the fitting problem can be solved by an eigensystem, which leads to a robust and computationally efficient method for paracatadioptric line estimation.
\end{abstract}

(c) 2005 Elsevier Inc. All rights reserved.

Keywords: Catadioptric; Paracatadioptric; Omnidirectional vision; Calibration; Lines; Line estimation

\section{Introduction}

The approach of combining mirrors with conventional cameras to enhance the sensor field of view is referred as catadioptric image formation. The use of catadioptric systems to achieve panoramic vision is simple and fast enabling the capture of dynamic scenes. The entire class of catadioptric configurations satisfying the single viewpoint constraint is derived in [1]. Panoramic central catadioptric systems can be built by combining a hyperbolic mirror with a perspective camera, and a parabolic mirror with an orthographic camera (paracatadioptric sensor).

\footnotetext{
* Corresponding author. Fax: +351 239406672.

E-mail addresses: jpbar@deec.uc.pt (J.P. Barreto), helder@isr.uc.pt (H. Araujo).

URL: www.deec.uc.pt/jpbar (J.P. Barreto).
}

The construction of the former requires a careful alignment between the mirror and the imaging device. The camera projection center must be positioned in the outer focus of the hyperbolic reflective surface. The paracatadioptric camera is easier to construct being broadly used in applications requiring omnidirectional vision [2-8]. In [9], Geyer and Daniilidis introduce for the first time a unifying theory for general central catadioptric image formation. A modified version of this mapping model is proposed in [10]. It is shown that central catadioptric projection is isomorphic to a projective mapping from a sphere, centered in the effective viewpoint, to a plane with a projection center on the perpendicular to the plane. For the particular case of paracatadioptric sensors the projection center lies on the sphere and the projective mapping is a stereographic projection. The plane and the final catadioptric image are related by a collineation depending on the mirror and 
camera intrinsic parameters. The system is calibrated when this collineation is known. It has already been proved that the central catadioptric projection of a line is a conic curve $[11,9,12]$, and that any central panoramic system can be fully calibrated from the image of three lines in general position [13]. However, since lines are mapped into conic curves which are only partially visible, the accurate estimation of catadioptric line images is far from being a trivial task $[14,15]$. The present work addresses the problem of accurately estimate paracatadioptric lines using image points.

Several authors have already proposed algorithms to calibrate a paracatadioptric camera [16-18]. The approach presented in [17] requires a sequence of paracatadioptric images. The system is calibrated using the consistency of pair wise tracked point features across the sequence, based on the characteristics of catadioptric imaging. In [18], the center and focal length are determined by fitting a circle to the image of the mirror boundary. The method is simple and can be easily automated, however it is not very accurate and requires the visibility of the mirror boundary. Its major drawback is that it is only applicable to the situation of a skewless camera with unitary aspect ratio. Geyer and Daniilidis [16] propose an algorithm to calibrate the sensor from an image of at least three lines. They present a closedform solution for focal length, image center, and aspect ratio for skewless cameras, and a polynomial root solution in the presence of skew. Certain properties of parabolic projection are used to get accurate line estimates, but the conic curves verifying these properties are not necessarily the paracatadioptric projection of lines. This has an impact on the global performance of the method as will be discussed in Section 5.

We derive for the first time the necessary and sufficient conditions that must be satisfied by a set of conic curves to be the paracatadioptric projection of lines. The derived conditions can be used to accurately estimate the line images using non-linear optimization. Moreover, if the system is skewless and the aspect ratio is known, then the line projections can be computed by solving an eigensystem. Given the image of at least three lines the paracatadioptric camera is easily calibrated using the geometric construction proposed in [13]. The calibration algorithm is evaluated using both synthetic and real images. The experimental results show that it out performs the method proposed in [16].

Additionally, we present a conic fitting algorithm that copes with the occlusion problem and accurately estimates the paracatadioptric image of single lines. The method is specific for line projections in parabolic systems and requires the sensor to be calibrated. We prove that a conic curve is the paracatadioptric image of a line if and only if the image of the circular points lie on the curve, and two specific points are harmonic conjugate with respect to the conic. The paracatadioptric camera maps lines in the scene into conic curves lying in a hyperplane in the space of all conics. Therefore, the line projection can be accurately determined by constraining the search space. The estimation algorithm is stable and computationally efficient because the fitting problem can be solved by an eigensystem. The proposed method is useful for many applications such as $3 \mathrm{D}$ reconstruction and visual control of motion using paracatadioptric images. Experimental results show that the approach is very robust and the estimation results are much better than the ones obtained by performing perspective rectification [4].

\section{Paracatadioptric projection of lines}

A general mapping model for central catadioptric systems has been introduced for the first time in [9]. This section briefly reviews the image formation model for the particular case of paracatadioptric cameras (for a detailed derivation see [10]). The equations for paracatadioptric line projection are derived, and it is shown that the image of a line is a conic curve $[9,11]$. The estimation of these conic curves is in general hard to accomplish due to partial occlusion. We compare and discuss the performance of five standard conic fitting methods.

\subsection{Paracatadioptric projection model}

Assume a paracatadioptric system combining a parabolic mirror, with latus rectum $4 p$, and an orthographic camera. The principal axis of the camera is aligned with the symmetry axis of the paraboloid. The paracatadioptric projection can be modeled by a stereographic projection from an unitary sphere, centered in the effective viewpoint, into a plane $\boldsymbol{\Pi}_{\infty}$ as shown in Fig. 1.

The mapping can be described as follows. Each visible scene point defines an oriented projective ray $\mathbf{x}=(x, y, z)^{t}$, joining the 3D point with the projection center $\mathbf{O}$. Consider
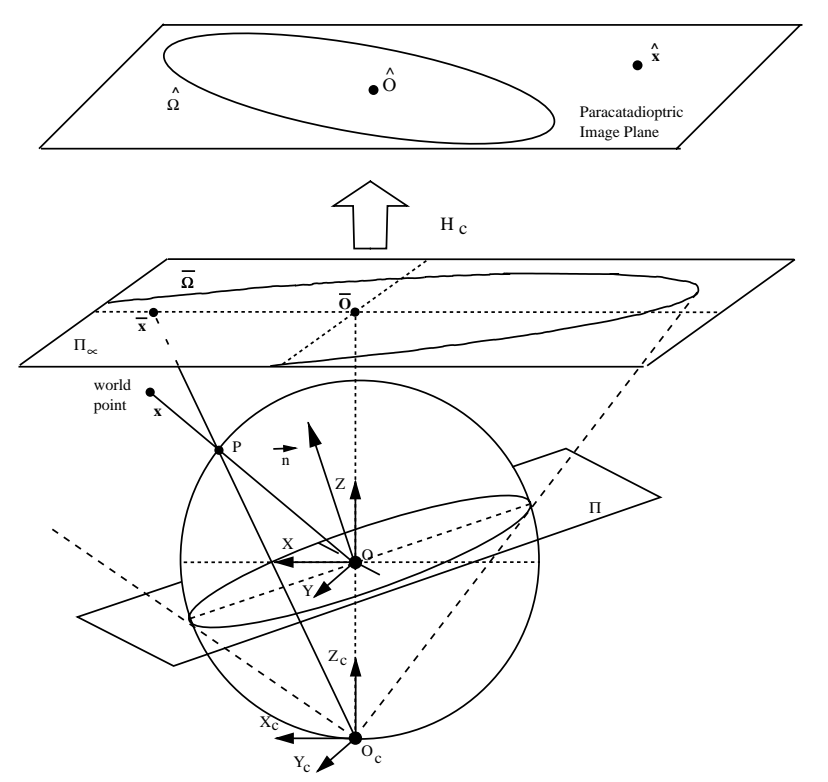

Fig. 1. Paracatadioptric projection model. The unitary sphere is centered at point $\mathbf{O}_{\mathbf{c}}$ and $\mathbf{P}$ is the point where the projective ray $\mathbf{x}$ intersects the sphere. The new projective point $\overline{\mathbf{x}}$ is defined by $\mathbf{O}_{\mathbf{c}}$ and $\mathbf{P}$. The paracatadioptric image point $\hat{\mathbf{x}}$ is related to $\overline{\mathbf{x}}$ by a projective transformation $\mathbf{H}_{\mathbf{c}}$. 
the south pole of the sphere $\mathbf{O}_{\mathbf{c}}$ with coordinates $(0,0,-1)^{t}$. To each $\mathbf{x}$ corresponds a projective ray $\overline{\mathbf{x}}$ going through $\mathbf{O}_{\mathbf{c}}$ and $\mathbf{P}$ (the intersection point of $\mathbf{x}$ and the sphere). This mapping $\hbar$ between projective points is non-linear as shown in Eq. (1). Function $\hbar$ is equivalent to projecting the scene on the unitary sphere, followed by a re-projection from the sphere to the plane $\boldsymbol{\Pi}_{\infty}$ with center $\mathbf{O}_{\mathbf{c}}$. Points in catadioptric image plane $\hat{\mathbf{x}}$ are obtained after a collineation $\mathbf{H}_{\mathbf{c}}$ of the $2 \mathrm{D}$ projective points $\overline{\mathbf{x}}$. Eq. (2) shows that $\mathbf{H}_{\mathbf{c}}$ is always an affine transformation depending on the camera intrinsic parameters $\mathbf{K}_{\mathbf{c}}$, and the latus rectum of the parabolic mirror.

$$
\begin{aligned}
& \hbar(\mathbf{x})=\left(x, y, z+\sqrt{x^{2}+y^{2}+z^{2}}\right)^{t}, \\
& \hat{\mathbf{x}}=\underbrace{\mathbf{K}_{\mathbf{c}}\left[\begin{array}{ccc}
2 p & 0 & 0 \\
0 & 2 p & 0 \\
0 & 0 & 1
\end{array}\right]}_{\mathbf{H}_{\mathbf{c}}} \overline{\mathbf{x}} .
\end{aligned}
$$

Consider plane $\boldsymbol{\Pi}=(\mathbf{n}, 0)^{t}$ going through the effective viewpoint $\mathbf{O}$ as depicted in Fig. $1\left(\mathbf{n}=\left(n_{x}, n_{y}, n_{z}\right)^{t}\right)$. The paracatadioptric image of any line lying in $\Pi$ is the conic curve $\hat{\Omega}$. The line in the scene is projected into a great circle on the sphere surface. This great circle is the curve of intersection of plane $\Pi$, containing both the line and the projection center $\mathbf{O}$, and the unit sphere. The projective rays $\overline{\mathbf{x}}$, joining $\mathbf{O}_{\mathbf{c}}$ with points on the great circle, form a central cone surface. The central cone, with vertex at $\mathbf{O}_{\mathbf{c}}$, projects into the conic $\bar{\Omega}$ in plane $\Pi_{\infty}$ Eq. (3). Since the image plane and $\boldsymbol{\Pi}_{\infty}$ are related by collineation $\mathbf{H}_{\mathbf{c}}$, the result of Eq. (4) comes in a straightforward manner.

$$
\begin{aligned}
& \bar{\Omega}=\left[\begin{array}{ccc}
-n_{z}^{2} & 0 & n_{x} n_{z} \\
0 & -n_{z}^{2} & n_{y} n_{z} \\
n_{x} n_{z} & n_{y} n_{z} & n_{z}^{2}
\end{array}\right], \\
& \hat{\mathbf{\Omega}}=\left[\begin{array}{lll}
a & b & d \\
b & c & e \\
d & e & f
\end{array}\right]=\mathbf{H}_{\mathbf{c}}^{-t} \overline{\mathbf{\Omega}} \mathbf{H}_{\mathbf{c}}^{-1} .
\end{aligned}
$$

The paracatadioptric system is calibrated whenever the collineation $\mathbf{H}_{\mathbf{c}}$ is known. Assume that the image center of the orthographic camera is $\mathbf{C}=\left(\mathbf{c}_{x}, c_{y}\right)^{t}$, and that $r_{c}^{2}, f_{o}$, and $s_{k}$ are respectively the aspect ratio, the focal length and the skew. Since the matrix of intrinsic parameters $\mathbf{K}_{\mathbf{c}}$ is upper triangular, then $\mathbf{H}_{\mathbf{c}}$ is always an affine transformation (Eq. (2)). Matrix $\mathbf{H}_{\mathbf{c}}$ is provided in Eq. (5) where $f_{\mathbf{c}}=2 f_{o}$ p is a measurement in pixels of the combined focal length of the camera and the mirror. As a final remark notice that since $\bar{\Omega}$ is a circle, then the conic curve $\hat{\Omega}$ is in general an ellipse (Eqs. (3) and (4)). Moreover, the paracatadioptric image of a line is a circle if and only if the orthographic camera has unitary aspect ratio $\left(r_{c}=1\right)$.

$$
\mathbf{H}_{\mathbf{c}}=\left[\begin{array}{ccc}
r_{c} f_{c} & s_{k} & c_{x} \\
0 & r_{c}^{-1} f_{c} & c_{y} \\
0 & 0 & 1
\end{array}\right] .
$$

\subsection{Estimation of paracatadioptric line images using standard conic fitting methods}

Eq. (4) shows that the paracatadioptric projection of a line is a conic curve which is parameterized by a $3 \times 3$ symmetric matrix $\hat{\Omega}$. Since a conic has five independent degrees of freedom (DOF), it can also be represented by a point $\hat{\boldsymbol{\omega}}$ in the 5D projective space $\wp^{5}$ (Eq. (6) [19]). Henceforth, we will assume both representations without distinction. The present section discusses the problem of fitting conic curves to image points to estimate the loci where lines are projected. A conic fitting algorithm determines the curve that best fits the data points according to a certain distance metric. We review some standard conic fitting methods [14,15] and evaluate their performance in estimating paracatadioptric line projections.

$\hat{\boldsymbol{\omega}}=(a, b, c, d, e, f)^{t}$.

The algebraic distance $\alpha_{i}$ from the image point $\hat{\mathbf{x}}_{\mathbf{i}}=\left(\hat{x}_{i}, \hat{y}_{i}\right)$ to the conic $\hat{\boldsymbol{\omega}}$ is defined by

$\alpha_{i}=a \hat{x}_{i}^{2}+2 b \hat{x}_{i} \hat{y}_{i}+c \hat{y}_{i}^{2}+2 d \hat{x}_{i}+2 e \hat{y}_{i}+f$.

Consider the set of $N$ distinct image points $\hat{\mathbf{x}}_{\mathbf{1}}, \hat{\mathbf{x}}_{\mathbf{2}} \ldots \hat{\mathbf{x}}_{\mathbf{N}}$. The corresponding vector of algebraic distances is $\left(\alpha_{1}, \alpha_{2}, \ldots, \alpha_{N}\right)^{t}=\mathbf{A} \hat{\boldsymbol{\omega}}$ where $\mathbf{A}$ is the following $N \times 6$ matrix

$\mathbf{A}=\left[\begin{array}{cccccc}\hat{x}_{1}^{2} & 2 \hat{x}_{1} \hat{y}_{1} & \hat{y}_{1}^{2} & 2 \hat{x}_{1} & 2 \hat{y}_{1} & 1 \\ \hat{x}_{2}^{2} & 2 \hat{x}_{2} \hat{y}_{2} & \hat{y}_{2}^{2} & 2 \hat{x}_{2} & 2 \hat{y}_{2} & 1 \\ \vdots & \vdots & \vdots & \vdots & \vdots & \vdots \\ \hat{x}_{N}^{2} & 2 \hat{x}_{N} \hat{y}_{N} & \hat{y}_{N}^{2} & 2 \hat{x}_{N} & 2 \hat{y}_{N} & 1\end{array}\right]$.

The sum of the square of the algebraic distances between the data points and the conic curve $\hat{\boldsymbol{\omega}}$ is

$\phi(\hat{\boldsymbol{\omega}})=\sum_{i=1}^{N} \alpha_{i}^{2}=\hat{\boldsymbol{\omega}}^{t} \mathbf{A}^{t} \mathbf{A} \hat{\boldsymbol{\omega}}$.

The algebraic distance $\alpha_{i}$ is zero whenever point $\hat{\mathbf{x}}_{\mathbf{i}}$ lies on the conic curve $\hat{\boldsymbol{\omega}}$. Thus, if $\hat{\mathbf{x}}_{\mathbf{1}}, \hat{\mathbf{x}}_{\mathbf{2}} \ldots \hat{\mathbf{x}}_{\mathbf{N}}$ are points on the conic then matrix $\mathbf{A}$ is rank deficient and $\hat{\boldsymbol{\omega}}$ is the respective right null space. In general the data points are noisy and $\mathbf{A}$ is full rank. In this case the square matrix $\mathbf{A}^{t} \mathbf{A}$ is non-singular and function $\phi(\hat{\boldsymbol{\omega}})$ has a single root $\hat{\boldsymbol{\omega}}=0$. There are several methods that minimize the objective function of Eq. (9) to fit a a conic to the data points. The solution $\hat{\boldsymbol{\omega}}=0$ is a global minimum of the function that must be avoided. The following algorithms differ in the way that the search space is constrained.

(1) The normal least squares (LMS) method determines the unit vector $\hat{\boldsymbol{\omega}}$ which minimizes the sum of the square distances to the data points. The cost function is $\phi_{\text {lms }}(\hat{\boldsymbol{\omega}}, \lambda)=\phi(\hat{\boldsymbol{\omega}})+\lambda\left(\hat{\boldsymbol{\omega}}^{t} \hat{\boldsymbol{\omega}}-1\right)$ where $\lambda$ is a Lagrange multiplier. The minimizer is the eigenvector corresponding to the smallest eigenvalue of matrix $\mathbf{A}^{t} \mathbf{A}[20]$. 
(2) The approximate mean square (AMS) metric was introduced by Taubin [21]. The AMS method minimizes the algebraic distance $\phi$ under the constraint $\hat{\boldsymbol{\omega}}^{t}\left(\mathbf{A}_{\mathbf{x}}^{t} \mathbf{A}_{\mathbf{x}}+\mathbf{A}_{\mathbf{y}}^{t} \mathbf{A}_{\mathbf{y}}\right) \hat{\boldsymbol{\omega}}=1$ where $\mathbf{A}_{\mathbf{x}}$ and $\mathbf{A}_{\mathbf{y}}$ are the partial derivatives of $\mathbf{A}$. In this case the minimizer is determined by solving the generalized eigensystem $\mathbf{A}^{t} \mathbf{A} \hat{\boldsymbol{\omega}}=\lambda\left(\mathbf{A}_{\mathbf{x}}^{t} \mathbf{A}_{\mathbf{x}}+\mathbf{A}_{\mathbf{y}}^{t} \mathbf{A}_{\mathbf{y}}\right) \hat{\boldsymbol{\omega}}$. The conic curve estimate is provided by the eigenvector corresponding to the smallest eigenvalue.

(3) In the method proposed by Fitzgibbon and Fisher (FF) the search space is constrained to the space of the ellipses [22]. The algorithm finds the ellipse $\hat{\omega}$ that minimizes the algebraic distance to the data points. The minimization can be stated as a generalized eigenproblem with a closed form solution.

The data points are usually obtained using an image processing algorithm (edge detection, contours, etc). It is reasonable to assume that the action of the noise is similar for all points and independent from one point to another [14]. Let the error in a generic point $\hat{\mathbf{x}}_{\mathbf{i}}=\left(\hat{x}_{i}, \hat{y}_{i}\right)^{t}$ be Gaussian with zero mean and covariance matrix $\sigma^{2} \mathbf{I}$ (I is the $2 \times 2$ identity matrix). Therefore, the noise variance in the algebraic distance is $\sigma_{i}^{2}=\nabla_{i} \sigma^{2}$, with $\nabla_{i}$ denoting the scalar Laplacian of $\alpha_{i}$ Eq. (7). The algorithms enumerated above provide the optimal solution in terms of the minimum covariance if and only if the $N$ equations $\alpha_{i}=0$ have the same variance and are statistically independent $[20,23]$. Since the Laplacian $\nabla_{i}$ is a function of the point coordinates, the variances $\sigma_{i}$ are not equal and the estimation results obtained using LMS, AMS, and FF are statistically biased [24]. This problem can be avoided by applying the following algorithm.

(4) The gradient weighted least square fitting algorithm (GRAD) divides the algebraic distances $\alpha_{i}$ by the scalar Laplacian $\nabla_{i}$ to normalize the variances $\sigma_{i}$. The corresponding objective function $\phi_{\text {grad }}$ is stated below. In this case, the solution can not be found solving an eigensystem and the problem has no longer a closed form solution. The minimization of $\phi_{\mathrm{grad}}$ must be performed using iterative gradient descent methods such as Gauss-Newton or Levenberg-Marquardt $[23,25]$.

$\phi_{\text {grad }}(\hat{\boldsymbol{\omega}})=\sum_{i=1}^{N} \frac{\alpha_{i}^{2}}{\nabla_{i}}=\sum_{i=1}^{N} \frac{\alpha_{i}^{2}}{\left(\frac{\partial \alpha_{i}}{\partial x_{i}}\right)^{2}+\left(\frac{\partial \alpha_{i}}{\partial y_{i}}\right)^{2}}$.

Methods based on algebraic distances, like the LMS, AMS, and FF algorithms, have a closed form solution because the estimation problem can be naturally solved by an eigensystem. However, each data point may contribute differently to the parameter estimation depending on its position on the conic. The problem of statistical bias is avoided in the GRAD method, but the objective function is not invariant under Euclidean transformations which causes undesired effects [14]. The following algorithm uses the geometric distance and the corresponding estimation results are invariant to rotation and translation.

(5) The ORTHO method minimizes the sum of the square of the orthogonal distances $\beta_{i}$ between the conic and the points (for further details about computing the orthogonal distances please consult the $[14,10])$. The objective function is $\phi_{\text {ortho }}$ and the minimum solution is determined using an iterative gradient descent method [25]:

$\phi_{\text {ortho }}(\hat{\boldsymbol{\omega}})=\sum_{i=1}^{N} \beta_{i}$.

Fig. 2A shows the robustness to noise of the described conic fitting methods. The performance suffers a graceful degradation in the presence of increasing noise. The GRAD and ORTHO algorithms are clearly more robust than the methods based on algebraic distances. Among the methods with closed form solution, the FF algorithm seems to be the most robust. In the experiment of Fig 2A the data points are distributed over the the entire conic. Fig. 2B shows the performance of the different algorithms when the conic curve is partially occluded. In this experiment the noise standard deviation is kept constant ( $\sigma=2$ pixel), and the samples are extracted from a partial arc with a certain amplitude. As expected, an increase in the angle of occlusion corresponds to a decrease in the performance of the estimators. All methods perform poorly when the amplitude of the occlusion is above $240^{\circ}$. None of the algorithms provide an useful estimate when the visible arc is less than $100^{\circ}$.

Section 2.1 proves that a line in the scene is projected into a conic locus in the paracatadioptric image plane. However, in most real images of lines, only a small conic arc is actually visible. Taking into account that in average the visible arc of a paracatadioptric line projection has an amplitude below $45^{\circ}$, we may conclude that these standard conic fitting techniques are unsuitable to estimate the conic locus where lines are mapped.

\section{Paracatadioptric camera calibration using lines}

Any central catadioptric system can be fully calibrated using the image of a minimum of three lines in general position $[13,10,16]$. In this work, we focus on the particular case of calibrating a paracatadioptric camera. Given the image of the lines, matrix $\mathbf{H}_{\mathbf{c}}$ (Eq. (5)) can be determined using the geometric construction proposed in [13]. This approach is straightforward whenever the conics corresponding to the line projections are accurately known. In the previous section we saw that, due to partial occlusion, the estimation of the conics using image points is hard to accomplish. To solve this problem, we derive for the first time the necessary and sufficient conditions that must be met by a set of conic 

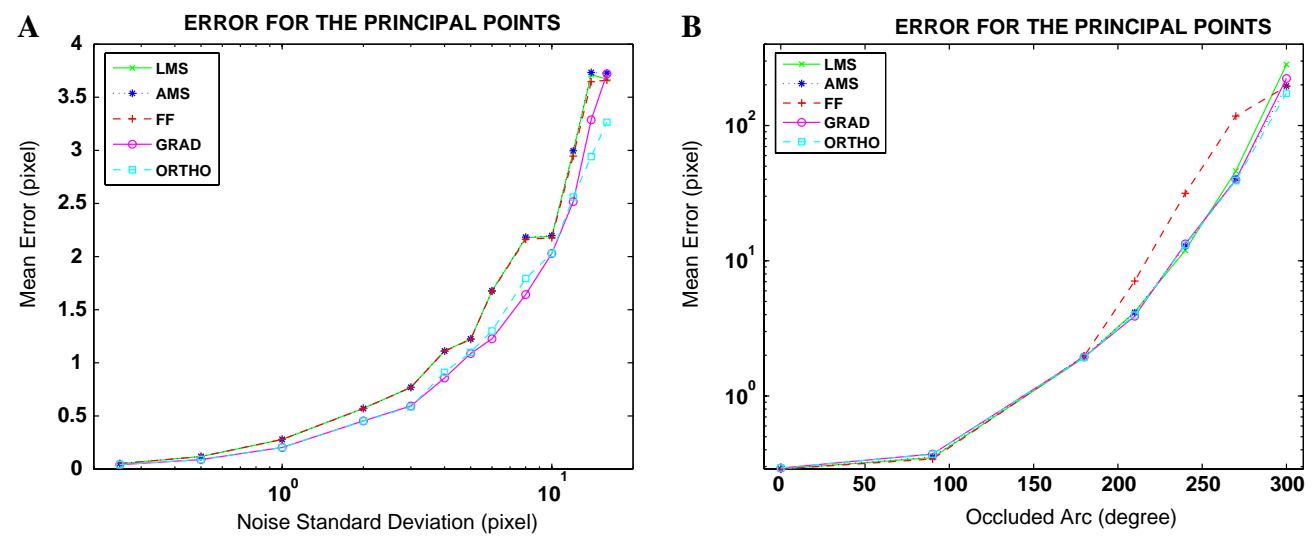

Fig. 2. Comparing the performance of standard conic fitting algorithms using synthetic data. The arc of the test conic is uniformly sampled by 100 points. Two-dimensional Gaussian noise with zero mean and standard deviation $\sigma$ is added to each sample point used in the estimation. The principal points of the estimated curve are compared with the ground truth and the mean error is computed over 100 runs of each experiment. (A) Robustness to noise. (B) Robustness to partial occlusion.

curves to be the paracatadioptric projection of lines. These conditions can be used to accurately estimate the line projections using non-linear minimization. Moreover, if the system is skewless and the aspect ratio is known, then the lines can be computed by solving an eigensystem.

\subsection{Calibration by geometric construction}

Table 1 summarizes the steps to calibrate a paracatadioptric system from the image of $K$ lines in general position. Fig. 3 is a scheme of the required geometric construction when the number of lines is minimum $(K=3)$. Assume that the $K$ lines are projected into the set of conic curves $\hat{\mathbf{\Omega}}_{\mathbf{1}}, \hat{\mathbf{\Omega}}_{\mathbf{2}}$, $\ldots \hat{\mathbf{\Omega}}_{K}$. Any two line projections $\hat{\boldsymbol{\Omega}}_{i}, \hat{\boldsymbol{\Omega}}_{j}$ intersect in two real points $\hat{\mathbf{B}}_{\mathrm{ij}}, \hat{\mathbf{F}}_{\mathrm{ij}}$. The image center $\hat{\mathbf{O}}$ must always lie on the line $\hat{\boldsymbol{\mu}}_{i j}$ defined by the intersection points $\hat{\mathbf{B}}_{\mathrm{ij}}$ and $\hat{\mathbf{F}}_{\mathrm{ij}}$ $[16,13]$. Consider the plane $\boldsymbol{\Pi}_{i}$ containing both the original 3D line and the effective viewpoint $\mathbf{O}$ (Fig. 1). If the line is projected into the conic $\hat{\mathbf{\Omega}}_{i}$, then the polar line $\hat{\boldsymbol{\pi}}_{\boldsymbol{i}}$ with respect to $\hat{\mathbf{O}}$ is the image of the vanishing line of $\boldsymbol{\Pi}_{i}$. Line $\hat{\pi}_{i}$ intersects the conic curve $\hat{\boldsymbol{\Omega}}_{i}$ in two points $\hat{\boldsymbol{I}}_{\mathbf{i}}$ and $\hat{\mathbf{J}}_{\mathbf{i}}$. It can be proved that these two points lie on the conic $\hat{\mathbf{\Omega}}_{\infty}$, which is the locus where the absolute conic is mapped by collineation $\mathbf{H}_{\mathbf{c}}$ [13]. Conic $\hat{\boldsymbol{\Omega}}_{\infty}$ can be estimated using the $K$ pairs of points $\hat{\mathbf{I}}_{\mathbf{i}}, \hat{\mathbf{J}}_{\mathbf{i}}(K \geqslant 3)$. Since $\mathbf{H}_{\mathbf{c}}$ is an upper triangular matrix Eq. (5) and $\hat{\mathbf{\Omega}}_{\infty}=\mathbf{H}_{\mathbf{c}}^{-t} \mathbf{H}_{\mathbf{c}}^{-1}$, then $\mathbf{H}_{\mathrm{c}}$ can be determined from the Cholesky decomposition of $\hat{\boldsymbol{\Omega}}_{\infty}$.

\subsection{Properties of a set of paracatadioptric line images}

A conic curve has five DOF and it can be represented either by a symmetric matrix $\hat{\Omega}$, or by a point $\hat{\omega}$ in $P^{5}$ (Eqs. (4) and (6)). Replacing $\overline{\boldsymbol{\Omega}}$ and $\mathbf{H}_{\mathbf{c}}$ in Eq. (4) by the results of Eqs. (3) and (5) yields

$$
\hat{\boldsymbol{\omega}}=\left[\begin{array}{l}
a \\
b \\
c \\
d \\
e \\
f
\end{array}\right]=\left[\begin{array}{c}
a \\
-\frac{r_{c} s_{k}}{f_{c}} a \\
\left(\frac{r_{c}^{2} s_{k}^{2}}{f_{c}^{2}}+r_{c}^{4}\right) a \\
d \\
e \\
-r_{c}^{2} f_{c}^{2} a-c_{x} d-c_{y} e
\end{array}\right]
$$

with

$$
\left\{\begin{array}{l}
a=-\frac{n_{z}^{2}}{r_{c}^{2} f_{c}^{2}}, \\
d=\frac{n_{x} n_{z}}{r_{c} f_{c}}-\frac{n_{z}^{2}\left(r_{c} s_{k} c_{y}-f_{c} c_{x}\right)}{r_{c}^{2} f_{c}^{3}}, \\
e=\frac{r_{c} n_{y} n_{z}}{f_{c}}+\frac{r_{c}^{2} n_{z}^{2} c_{y}-s_{k} n_{x} n_{z}}{f_{c}^{2}}+\frac{s_{k} n_{z}^{2}\left(r_{c} s_{k} c_{y}-f_{c} c_{x}\right)}{r_{c} f_{c}^{4}} .
\end{array}\right.
$$

The paracatadioptric image of a line depends both on the system intrinsic parameters and the orientation of the $3 \mathrm{D}$ plane $\Pi$ (Fig. 1). Assume $K$ lines in the scene that are projected in the set of conic curves $\hat{\omega}_{i}$

$\hat{\omega}_{i}=\left(a_{i}, b_{i}, c_{i}, d_{i}, e_{i}, f_{i}\right)^{t}, \quad i=1,2,3, \ldots, K$.

Table 1

Calibration of a paracatadioptric system using $K$ lines $(K \geqslant 3)$.

\begin{tabular}{ll}
\hline Step 1 & Determine the catadioptric line images $\hat{\mathbf{\Omega}}_{\boldsymbol{i}}$ for $i=1,2,3, \ldots, K$ \\
Step 2 & For each pair of conics $\hat{\mathbf{\Omega}}_{\boldsymbol{i}}, \hat{\mathbf{\Omega}}_{\mathbf{j}}$, compute the intersection points $\hat{\mathbf{F}}_{\mathbf{i j}}, \hat{\mathbf{B}}_{\mathbf{i j}}$, and determine the corresponding line $\hat{\boldsymbol{\mu}}_{\boldsymbol{i j}}=\hat{\mathbf{F}}_{\mathbf{i j}} \wedge \hat{\mathbf{B}}_{\mathbf{i j}}$ \\
Step 3 & Estimate the image center $\mathbf{\mathbf { O }}$ which is the intersection point of lines $\hat{\boldsymbol{\mu}}_{\mathbf{i j}}$ \\
Step 4 & For each conic $\hat{\mathbf{\Omega}}_{\boldsymbol{i}}$ compute the polar line $\hat{\boldsymbol{\pi}}_{\boldsymbol{i}}$ of the image center $\hat{\mathbf{O}}(i=1,2,3, \ldots, K)$. \\
Step 5 & For each conic curve obtain the points $\hat{\mathbf{I}}_{\mathbf{i}}$ and $\hat{\mathbf{J}}_{\mathbf{i}}$ where line $\hat{\pi}_{\mathbf{i}}$ intersects $\hat{\mathbf{\Omega}}_{\boldsymbol{i}}(i=1,2,3, \ldots, K)$ \\
Step 6 & Estimate the conic $\hat{\mathbf{\Omega}}_{\infty}$ going through points $\hat{\mathbf{I}}_{\mathbf{i}}, \hat{\mathbf{J}}_{\mathbf{i}}(i=1,2,3, \ldots, K)$ \\
Step 7 & Perform the Cholesky decomposition of $\hat{\mathbf{\Omega}}_{\infty}$ to estimate matrix $\mathbf{H}_{\mathbf{c}}$
\end{tabular}

For a detailed proof of the method please consult $[13,10]$. 


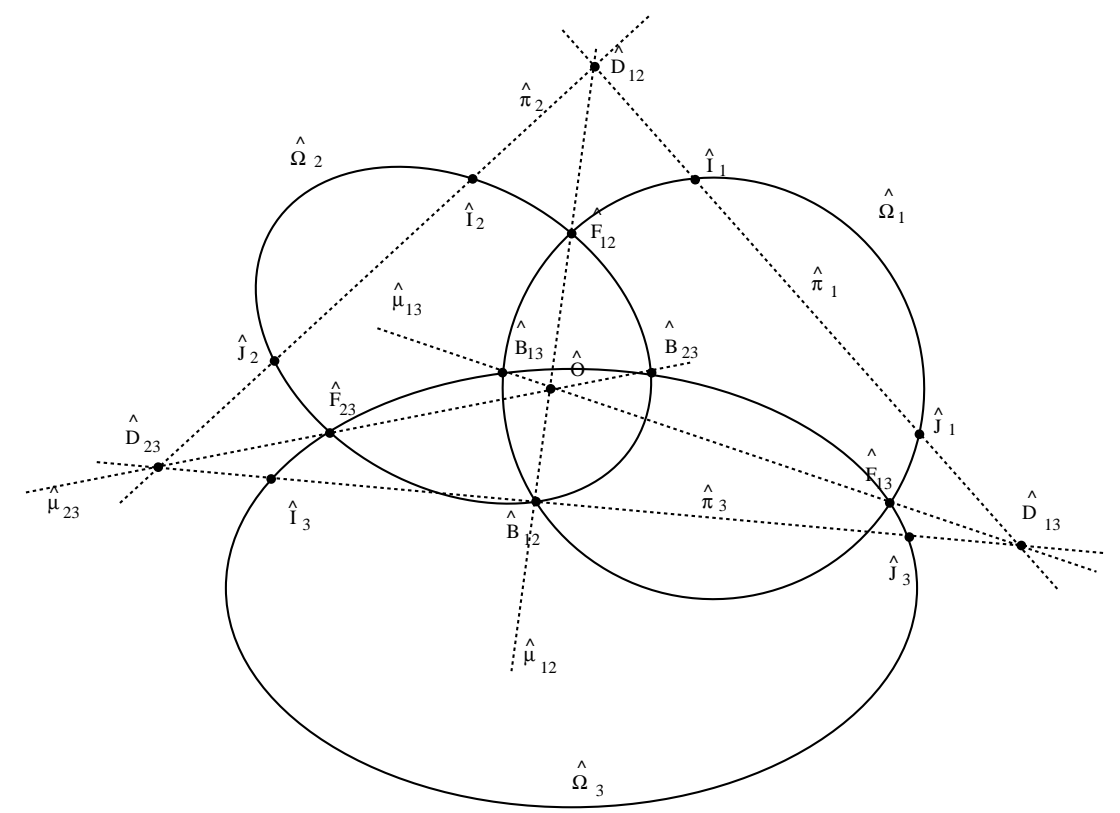

Fig. 3. Geometric construction to calibrate a paracatadioptric camera from the projection of three lines in general position. The lines are mapped into the conics $\boldsymbol{\Omega}_{1}, \boldsymbol{\Omega}_{2}$, and $\boldsymbol{\Omega}_{3}$ that must be accurately determined.

From Eq. (10) follows that

$\frac{b_{1}}{a_{1}}=\frac{b_{2}}{a_{2}}=\frac{b_{3}}{a_{3}}=\cdots=\frac{b_{K}}{a_{K}}=-\frac{r_{c} s_{k}}{f_{c}}$

$\frac{c_{1}}{a_{1}}=\frac{c_{2}}{a_{2}}=\frac{c_{3}}{a_{3}}=\cdots=\frac{c_{K}}{a_{K}}=\frac{r_{c}^{2} s_{k}^{2}}{f_{c}^{2}}+r_{c}^{4}$.

with

$\left\{\begin{array}{l}a=-\frac{n_{z}^{2}}{r_{c}^{2} f_{c}^{2}}, \\ d=\frac{n_{x} n_{z}}{r_{c} c_{c}}-\frac{n_{z}^{2}\left(r_{c} s_{k} c_{y}-f_{c} c_{x}\right)}{r_{c}^{2} f_{c}^{3}}, \\ e=\frac{r_{c} n_{y} n_{z}}{f_{c}}+\frac{r_{c}^{2} n_{z}^{2} c_{y}-s_{k} n_{x} n_{z}}{f_{c}^{2}}+\frac{s_{k} n_{z}^{2}\left(r_{c} s_{k} c_{c}-f_{c} c_{x}\right)}{r_{c} f_{c}^{4}} .\end{array}\right.$

From the first expression it follows that $\eta_{i}=0$ for $i=2,3, \ldots, K$, with $\eta_{i}$ obtained from Eq. (12). Moreover, using the second expression in a similar manner, one obtains $\chi_{i}=0$ for $i=2,3, \ldots, K$ with $\chi_{i}$ given by Eq. (13).

$\eta_{i}=a_{1} b_{i}-a_{i} b_{1}, \quad i=2,3, \ldots, K$,

$\chi_{i}=a_{1} c_{i}-a_{i} c_{1}, \quad i=2,3, \ldots, K$.

From the result of Eq. (10) it follows that each line projection $\hat{\omega}_{i}$ verifies $r_{c}^{2} f_{c}^{2} a_{i}+c_{x} d_{i}+c_{y} e_{i}+f_{i}=0$. Consider the first three elements $\hat{\omega}_{1}, \hat{\omega}_{2}$, and $\hat{\omega}_{3}$, of the set of line projections. The parameters $r_{c}^{2} f_{c}^{2}, \mathbf{c}_{x}$, and $c_{y}$ can be estimated from

$\left[\begin{array}{c}r_{c}^{2} f_{c}^{2} \\ c_{x} \\ c_{y}\end{array}\right]=-\underbrace{\left[\begin{array}{lll}a_{1} & d_{1} & e_{1} \\ a_{2} & d_{2} & e_{2} \\ a_{3} & d_{3} & e_{3}\end{array}\right]}_{\boldsymbol{\Phi}^{-1}} \underbrace{\left[\begin{array}{l}f_{1} \\ f_{2} \\ f_{3}\end{array}\right]}_{\Gamma}$.

Therefore each conic curve $\hat{\omega}_{i}$, with $i=4, \ldots, K$, must verify the constraint $v_{i}=0$ Eq. (14). $v_{i}=\left[\begin{array}{llll}a_{i} & d_{i} & e_{i} & f_{i}\end{array}\right] \cdot\left[\begin{array}{c}-\boldsymbol{\Phi}^{-1} \boldsymbol{\Gamma} \\ 1\end{array}\right], \quad i=4, \ldots, K$.

If a set of $K$ conic curves corresponds to the paracatadioptric projection of $K$ lines, then $\eta_{i}, \chi_{i}$, and $v_{i}$, provided in Eqs. (12)-(14), must be equal to zero. We have derived $3 K-5$ independent conditions that are necessary for a set of $K$ conic curves to be the paracatadioptric projection of a set of $K$ lines in the scene. However it has not been proved that these conditions are also sufficient. By sufficient we mean that, if a certain set of conic curves satisfies these conditions then it can be the paracatadioptric projection of a set of lines. Consider the uncalibrated image of $K$ lines that are mapped in the same number of conics. Since each conic has five DOF then a set of $K$ conics has a total of $5 K$ DOF. Each line introduces two unknowns (DOF), which correspond to the orientation of the associated plane $\boldsymbol{\Pi}$ (see Fig. 1). Moreover, the five parameters of matrix $\mathbf{H}_{\mathbf{c}}$ are also unknown Eq. (5). Therefore, we have a total of $2 K+5$ unknowns (DOF). Since $5 K>2 K+5$ then it is obvious that there are sets of conic curves that can never be the paracatadioptric projection of lines. The conics that can correspond to the image of the lines lie in a subspace of dimension $2 K+5$. This means that there are $3 K-5$ independent constraints, which proves the sufficiency of the conditions derived above.

\subsection{Estimation of a set of $K$ paracatadioptric line images}

Consider the image of $K$ lines acquired by a non-calibrated paracatadioptric sensor. The lines are mapped into a set of $K$ conic curves $\hat{\omega}_{i}$ Eq. (11) that we aim to estimate. To each curve $\hat{\boldsymbol{\omega}}_{i}$ corresponds a set of image points $\hat{\mathbf{x}}_{\mathbf{i}}^{\mathrm{i}}$, with 
$j=1,2, \ldots, N_{i}$ and $N_{i} \geqslant 5$. The data points are used to build a matrix $\mathbf{B}$ with the sub-matrices $\mathbf{A}_{i}$ similar to matrix A of Eq. (8).

$$
\mathbf{B}=\left[\begin{array}{ccccc}
\mathbf{A}_{1} & 0 & 0 & \cdots & 0 \\
0 & \mathbf{A}_{2} & 0 & \cdots & 0 \\
0 & 0 & \mathbf{A}_{3} & \cdots & 0 \\
\vdots & \vdots & \vdots & \ddots & \vdots \\
0 & 0 & 0 & \cdots & \mathbf{A}_{\mathbf{K}}
\end{array}\right] .
$$

The sum of the square of the algebraic distances between the data points and the set of conics $\hat{\omega}_{i}$ is provided by function $\epsilon(\mathbf{p})$ with $\mathbf{p}=\left(\hat{\boldsymbol{\omega}}_{1}^{t}, \hat{\boldsymbol{\omega}}_{2}^{t}, \ldots, \hat{\boldsymbol{\omega}}_{K}^{t}\right)^{t}$

$\epsilon(\mathbf{p})=\mathbf{p}^{t} \mathbf{B}^{t} \mathbf{B} \mathbf{p}$.

As discussed in Section 2.2, the set of conic curves can be estimated by finding the minimum of function $\epsilon$. The problem is that in general the conic curves corresponding to the paracatadioptric projection of lines are strongly occluded in the image. The standard conic fitting techniques do not work properly in these conditions since the data points do not provide enough information to correctly estimate the conics. We propose to use the derived necessary and sufficient conditions to constrain the search space and improve the estimation results.

\subsubsection{General case}

In general nothing is known about the system calibration. The skew $s_{k}$ can be non-null and the aspect ratio $r_{c}^{2}$ can be different from one (Eq. (5)). According to Section 3.2 , a set of $K$ conic curves $\mathbf{p}=\left(\hat{\boldsymbol{\omega}}_{1}^{t}, \hat{\boldsymbol{\omega}}_{2}^{t}, \ldots, \hat{\boldsymbol{\omega}}_{K}^{t}\right)^{t}$ is the paracatadioptric projection of a set of lines if and only if the constraints of Eqs. (12)-(14) are verified. We aim to determine the $6 k \times 1$ vector $\mathbf{p}$, that minimizes function $\epsilon$, and verifies $\eta_{i}=0, \chi_{i}=0$ and $v_{i}=0$. The constraints can be introduced using a Lagrange multiplier $\lambda$, and the objective function is

$\epsilon_{g}(\mathbf{p}, \lambda)=\epsilon(\mathbf{p})+\lambda\left(\sum_{i=2}^{K} \eta_{i}^{2}+\sum_{i=2}^{K} \chi_{i}^{2}+\sum_{i=4}^{K} v_{i}^{2}\right)$.

The minimization of $\epsilon_{g}$ can be stated as a non-linear least squares problem, and the solution found using GaussNewton or Levenberg-Marquardt algorithms [23,25].

\subsubsection{Skewless images with known aspect ratio}

Assume that the orthographic camera is skewless and that the aspect ratio $r_{c}^{2}$ is known. Replacing $s_{k}$ by 0 in Eq. (10) yields $b=0$ and $c=r_{c}^{4} a$. The constraints $\eta_{i}=0$ and $\chi_{i}=0$ for $i=2, \ldots, K$, become $b_{i}=0$ and $c_{i}-r_{c}^{4} a_{i}=0$ for $i=1, \ldots, K$. There are two additional constraints because two of the calibration parameters are known. A new objective function $\epsilon_{s}$ is derived from Eq. (17)

$$
\epsilon_{s}(\mathbf{p}, \lambda)=\epsilon(\mathbf{p})+\lambda\left(\sum_{i=1}^{K} b_{i}^{2}+\sum_{i=1}^{K}\left(c_{i}-r_{c}^{4} a_{i}\right)^{2}+\sum_{i=4}^{K} v_{i}^{2}\right) .
$$

Each curve in the set has a matrix $\mathbf{A}_{\mathbf{i}}$ associated with it (Eq. (8)). Since the camera is skewless and the aspect ratio is known, then $b_{i}=0$ and $c_{i}=r_{c}^{4} a_{i}$. Omitting the second column of $\mathbf{A}_{\mathbf{i}}$ and adding the third column, multiplied by $r_{c}^{4}$, to the first column yields

$\dot{\mathbf{A}}_{\mathbf{i}}=\left[\begin{array}{cccc}\hat{x}_{1}^{2}+r_{c}^{4} \hat{y}_{1}^{2} & 2 \hat{x}_{1} & 2 \hat{y}_{1} & 1 \\ \hat{x}_{2}^{2}+r_{c}^{4} \hat{y}_{2}^{2} & 2 \hat{x}_{2} & 2 \hat{y}_{2} & 1 \\ \vdots & \vdots & \vdots & \vdots \\ \hat{x}_{N_{i}}^{2}+r_{c}^{4} \hat{y}_{N_{i}}^{2} & 2 \hat{x}_{N_{i}} & 2 \hat{y}_{N_{i}} & 1\end{array}\right]$.

The sum of the squares of the algebraic distances between the conic curve and the data points is $\dot{\phi}_{i}=\dot{\omega}_{i}^{t} \dot{\mathbf{A}}_{\mathbf{i}}^{t} \dot{\mathrm{A}}_{i} \dot{\omega}_{i}$ with $\dot{\omega}_{i}=\left(a_{i}, d_{i}, e_{i}, f_{i}\right)^{t}$. By combining the $K$ conics we obtain the sum of squares of the algebraic distances between the set of curves and the data points. This sum is given by the function

$\dot{\epsilon}(\mathbf{q})=\mathbf{q}^{t} \dot{\mathbf{B}}^{t} \dot{\mathbf{B}} \mathbf{q}$,

where matrix $\dot{\mathbf{B}}$ is obtained by replacing $\mathbf{A}_{\mathbf{i}}$ by $\dot{\mathbf{A}}_{\mathbf{i}}$ in Eq. (15), and $\mathbf{q}=\left(\dot{\boldsymbol{\omega}}_{1}^{t}, \dot{\boldsymbol{\omega}}_{\mathbf{2}}^{t}, \ldots \dot{\boldsymbol{\omega}}_{K}^{t}\right)^{t}$. Since $\dot{\mathbf{B}}$ implicitly encodes the constraints $b_{i}=0$ and $c_{i}-r_{c}^{4} a_{i}=0$, the objective function $\epsilon_{s}$ of Eq. (18) can be rewritten as

$\dot{\epsilon}_{s}(\mathbf{q}, \lambda)=\dot{\epsilon}(\mathbf{q})+\lambda \sum_{i=4}^{K} v_{i}^{2}$.

As discussed in Section 2.2, the eigenvector corresponding to the smallest eigenvalue of matrix $\dot{\mathbf{B}}^{t} \dot{\mathbf{B}}$ is the solution $\mathbf{q}$ that minimizes $\dot{\epsilon}$ under the constraint $\mathbf{q}^{t} \mathbf{q}=1$ [20]. If $K=3$ then the second term of Eq. 20 disappears and the minimization problem has a closed form solution. If $K>3$ then the minimum of function $\dot{\epsilon}_{s}$ must be found using an iterative gradient descent method. In this situation the eigenvector solution can be used as an initial estimate.

\section{Direct least square fitting of paracatadioptric line images}

The paracatadioptric image of a line is a conic curve that in general is hard to estimate due to partial occlusion. Section 3 shows that a set of conic curves must verify certain properties to be a coherent paracatadioptric projection of a set of lines. The conditions of Eqs. (13)-(15) depend neither on the system calibration nor on the $3 \mathrm{D}$ position of the lines. These conditions are used to constrain the search space and estimate the set of conic curves in the uncalibrated image. The set of line images is used to calibrate the paracatadioptric camera using the geometric construction presented in $[13,10]$.

While Section 3 focuses in the estimation of a set of line projections in an uncalibrated paracatadioptric image, the present section discusses the problem of determining the projection of a single line in a calibrated paracatadioptric view. Considering the space of all conic curves, it is proved that the paracatadioptric projection of any line lies in a hyperplane defined by the calibration parameters. The line image can be estimated by fitting the data points by a conic 
lying in this linear subspace. The proposed approach is computationally efficient because the fitting problem can be solved by an eigensystem.

\subsection{The necessary and sufficient conditions}

The scheme of Fig. 1 shows the paracatadioptric projection of a line. The line lies in a plane $\boldsymbol{\Pi}$ going through the effective viewpoint $\mathbf{O}$. The mapping from the sphere into the plane $\boldsymbol{\Pi}_{\infty}$ is a stereographic projection. Plane $\boldsymbol{\Pi}$ intersects the sphere in a great circle that is projected into a circle $\overline{\mathbf{\Omega}}$ Eq. 3. Points in plane $\boldsymbol{\Pi}_{\infty}$ are mapped into points in the image by an affine transformation $\mathbf{H}_{\mathbf{c}}$ Eq. (5). Since an affine transformation does not change the type of conic, then the paracatadioptric projection of a line $\hat{\boldsymbol{\Omega}}$ is always a circle/ellipse Eq. (4). Consider the following points lying in plane $\boldsymbol{\Pi}_{\infty}$ :

$\overline{\mathbf{I}}_{\infty}=(1, i, 0)^{t}$,

$\overline{\mathbf{J}}_{\infty}=(1,-i, 0)^{t}$,

$\overline{\mathbf{G}}_{\infty}=(1,0,-i)^{t}$,

$\overline{\mathbf{H}}_{\infty}=(1,0, i)^{t}$.

Assume that the paracatadioptric system is calibrated and the affine transformation $\mathbf{H}_{\mathbf{c}}$ is known. The above points are mapped in the paracatadioptric image plane in points:

$\hat{\mathbf{I}}_{\infty}=\mathbf{H}_{\mathbf{c}} \overline{\mathbf{I}}_{\infty}=\left(i_{x}, i_{y}, i_{z}\right)^{t}$,

$\hat{\mathbf{J}}_{\infty}=\mathbf{H}_{\mathbf{c}} \overline{\mathbf{J}}_{\infty}=\left(j_{x}, j_{y}, j_{z}\right)^{t}$,

$\hat{\mathbf{G}}_{\infty}=\mathbf{H}_{\mathbf{c}} \overline{\mathbf{G}}_{\infty}=\left(g_{x}, g_{y}, g_{z}\right)^{t}$,

$\hat{\mathbf{H}}_{\infty}=\mathbf{H}_{\mathbf{c}} \overline{\mathbf{H}}_{\infty}=\left(h_{x}, h_{y}, h_{z}\right)^{t}$.

Using these points we can state the following proposition

Proposition 1. A conic curve $\hat{\boldsymbol{\Omega}}$ is the paracatadioptric image of a line in the scene if and only if it contains points $\hat{\mathbf{I}}_{\infty}$ and $\hat{\mathbf{J}}_{\infty}\left(\hat{\mathbf{I}}_{\infty}^{t} \hat{\mathbf{\Omega}} \hat{\mathbf{I}}_{\infty}=0, \hat{\mathbf{J}}_{\infty}^{t} \hat{\mathbf{\Omega}} \hat{\mathbf{J}}_{\infty}=0\right)$, and points $\hat{\mathbf{G}}_{\infty}, \hat{\mathbf{H}}_{\infty}$ are conjugate with respect to $\hat{\mathbf{\Omega}}\left(\hat{\mathbf{G}}_{\infty}^{t} \hat{\mathbf{\Omega}} \hat{\mathbf{H}}_{\infty}=0\right)$.

Proof. Consider the conic curve $\overline{\mathbf{\Omega}}=\mathbf{H}_{\mathbf{c}}^{t} \hat{\mathbf{\Omega}} \mathbf{H}_{\mathbf{c}}$, lying in plane $\boldsymbol{\Pi}_{\infty}$ (Fig. 1). Conic $\overline{\boldsymbol{\Omega}}$ is a function of the normal $\mathbf{n}$ to the plane $\boldsymbol{\Pi}$ Eq. (3). Since $\overline{\boldsymbol{\Omega}}$ is a circle, then it must go through the circular points $\overline{\mathbf{I}}_{\infty}$ and $\overline{\mathbf{J}}_{\infty}$. Moreover, from Eq. (3), it follows that points $\overline{\mathbf{G}}_{\infty}$ and $\overline{\mathbf{H}}_{\infty}$ are always harmonic conjugate with respect to conic $\overline{\mathbf{\Omega}}$. Remark that these properties are independent of the orientation $\mathbf{n}$ of plane $\boldsymbol{\Pi}$. Since collineation $\mathbf{H}_{\mathbf{c}}$ preserves incidence and harmonic relations, then conic $\hat{\boldsymbol{\Omega}}$ must satisfy $\hat{\mathbf{I}}^{t} \hat{\mathbf{\Omega}} \hat{\mathbf{I}}=0, \hat{\mathbf{J}}^{t} \hat{\mathbf{\Omega}} \hat{\mathbf{J}}=0$ and $\hat{\mathbf{G}}^{t} \hat{\mathbf{\Omega}} \hat{\mathbf{H}}=0$. The conditions derived are necessary, nevertheless it is not clear that they are sufficient. By sufficient we mean that if a conic curve verifies these three constraints, then it is the locus where a certain line in the scene is projected. By neglecting the scale factor, the conic curve $\overline{\mathbf{\Omega}}$ of Eq. (3) is a function of two independent parameters. These two degrees of freedom (DOF) are associated with the pose of plane $\Pi$ containing both the original line and the effective viewpoint (Fig. 1). Since in general a conic curve has five DOF, then we must be able to find three, and no more than three, independent constraints. This proves the sufficiency of the statement.

The established proposition has an interesting geometric interpretation. It has already been stated that any conic curve $\hat{\boldsymbol{\Omega}}$ can be parameterized by a point $\hat{\boldsymbol{\omega}}$ in $\wp^{5}$ (Eq. (6)). Consider the $3 \times 6$ matrix $\mathbf{Y}$ provided in Eq. (22). The matrix is defined by points $\hat{\mathbf{I}}_{\infty}, \hat{\mathbf{J}}_{\infty}, \hat{\mathbf{G}}_{\infty}$, and $\hat{\mathbf{H}}_{\infty}$ Eq. (21). According to Proposition 1, a conic curve is the paracatadioptric image of a line if and only if the corresponding $6 \times 1$ vector $\hat{\boldsymbol{\omega}}$ lies in the null space of $\boldsymbol{\Upsilon}(\boldsymbol{\Upsilon} \hat{\boldsymbol{\omega}}=0)$. This means that all points $\hat{\omega}$, parameterizing the paracatadioptric projection of a line, must lie in a certain plane in the five-dimensional projective space. This plane is defined by matrix $\mathbf{Y}$ and depends exclusively on the camera calibration.

$$
\mathbf{r}=\left[\begin{array}{llllll}
i_{x}^{2} & 2 i_{x} i_{y} & i_{y}^{2} & 2 i_{x} i_{z} & 2 i_{y} i_{z} & i_{z}^{2} \\
j_{x}^{2} & 2 j_{x} j_{y} & j_{y}^{2} & 2 j_{x} j_{z} & 2 j_{y} j_{z} & j_{z}^{2} \\
g_{x} h_{x} & g_{x} g_{y}+h_{x} h_{y} & g_{y} h_{y} & g_{x} g_{z}+h_{x} h_{z} & g_{y} g_{z}+h_{y} h_{z} & g_{z} h_{z}
\end{array}\right] .
$$

\subsection{The fitting algorithm}

Consider a set of image points $\hat{\mathbf{x}}_{\mathbf{i}}=\left(\hat{x}_{i}, \hat{y}_{i}\right)^{t}$ with $i=1,2, \ldots, N$. Our goal is to fit a conic curve $\hat{\boldsymbol{\omega}}$, corresponding to the paracatadioptric projection of a line, to the set of data points. From Section 2.2 it follows that the sum of the squares of the algebraic distances between the curve and the image points is $\phi(\hat{\boldsymbol{\omega}})=\hat{\boldsymbol{\omega}}^{t} \mathbf{A}^{t} \mathbf{A} \hat{\boldsymbol{\omega}}$ (matrix $\mathbf{A}$ is provided in Eq. (8)).

Since a conic has five DOF, the space of all conics has five dimensions. The standard conic fitting algorithms of Section 2.2 search the entire space for the conic that best fits the data points. However, and according to Proposition 1 , not all conics can be the paracatadioptric projection of a line. The line projection $\hat{\boldsymbol{\omega}}$ must be in the null space of matrix $\mathbf{\Upsilon}$ Eq. (22). The null space of $\mathbf{\Upsilon}$ is a linear subspace (hyperplane) in the space of all conic curves. Our approach fits the data by the conic curve in this hyperplane that minimizes the algebraic distance to the image points. Consider the singular value decomposition of matrix $\mathbf{r}$.

$\mathbf{Y}=\mathbf{U S V}^{t}$.

Matrices $\mathbf{U}, \mathbf{S}$, and $\mathbf{V}$ have respectively dimension $3 \times 3$, $3 \times 6$, and $6 \times 6$. Matrix $\mathbf{V}$ is full rank and orthonormal $\left(\mathbf{V}^{-1}=\mathbf{V}^{t}\right)$. The three last columns of $\mathbf{V}$ are an orthonormal basis of the null space of $\mathbf{Y}[23,26]$. Consider the change on the base of representation $\hat{\boldsymbol{\omega}}_{\boldsymbol{v}}=\mathbf{V} \hat{\boldsymbol{\omega}}$. If $\hat{\boldsymbol{\omega}}$ belongs to the null space of matrix $\mathbf{r}$, then the corresponding $\hat{\omega}_{v}$ has the following structure:

$\hat{\boldsymbol{\omega}}_{v}=(0,0,0, \underbrace{d_{v}, e_{v}, f_{v}}_{\rho})^{t}$. 
Rewriting the algebraic distance of Eq. (9) in terms of the new coordinates one obtains $\phi=\hat{\boldsymbol{\omega}}_{v}^{t} \mathbf{V} \mathbf{A}^{t} \mathbf{A} \mathbf{V}^{t} \hat{\boldsymbol{\omega}}_{\boldsymbol{v}}$. Taking into account the structure of $\hat{\boldsymbol{\omega}}_{\boldsymbol{v}}$ Eq. (23), the algebraic distance becomes $\phi=\boldsymbol{\rho}^{t} \boldsymbol{\Lambda}^{t} \boldsymbol{\Lambda} \boldsymbol{\rho}$ with $\boldsymbol{\Lambda}$ the bottom right $3 \times 3$ sub matrix of $\mathbf{V} \mathbf{A}^{t} \mathbf{A} \mathbf{V}^{t}$. We aim to determine the solution $\boldsymbol{\rho}$ which minimizes the algebraic distance $\phi$ under the constraint $\boldsymbol{\rho}^{t} \boldsymbol{\rho}=1$. The corresponding objective function is

$\psi(\boldsymbol{\rho}, \lambda)=\boldsymbol{\rho}^{t} \boldsymbol{\Lambda}^{t} \boldsymbol{\Lambda} \boldsymbol{\rho}+\lambda\left(\boldsymbol{\rho}^{t} \boldsymbol{\rho}-1\right)$.

The minimum of the objective function $\psi$ is the eigenvector of matrix $\boldsymbol{\Lambda}^{t} \boldsymbol{\Lambda}$ corresponding to the smallest eigenvalue. The final conic $\hat{\omega}$ is computed by replacing $\rho$ in Eq. (24) and making $\hat{\boldsymbol{\omega}}=\mathbf{V}^{t} \hat{\boldsymbol{\omega}}_{\boldsymbol{v}}$.

\section{Performance evaluation using synthetic data}

The performance of the proposed algorithms is evaluated using synthetically generated images. This section starts by introducing the scheme to generate synthetic data. In Section 5.2 a skewless system with unitary aspect ratio is calibrated using just three lines $(K=3)$. It is shown that our approach outperforms the method proposed in [16]. The calibration of a general paracatadioptric system (unknown skew and aspect ratio) is discussed in Section 5.3. The section ends with the performance evaluation of the fitting method to estimate the conic locus where a line is projected in a calibrated paracatadioptric image.

\subsection{Simulation scheme}

Assume a paracatadioptric camera with a field of view (FOV) of $180^{\circ}$, corresponding to a full hemisphere, and predefined intrinsic parameters. The image of a set of $K$ lines is generated as follows. As depicted in Fig. 1, to each line in the scene corresponds a plane $\boldsymbol{\Pi}$ with normal $\mathbf{n}$. The $K$ normals are unitary and randomly chosen from an uniform distribution in the sphere. Each normal defines a plane that intersects the unit sphere on a great circle. Notice that half of the great circle is within the camera field of view (the $\mathrm{FOV}$ is $180^{\circ}$ ). An angle $\theta$, less than or equal to the FOV, is chosen to be the amplitude of the arc that is actually visible in the paracatadioptric image. The arc is randomly and uniformly positioned along the part of the great circle which is within the FOV. The visible arc is uniformly sampled by a fixed number $N$ of sample points. To each sample point corresponds a projective ray $\mathbf{x}$. The sample rays are projected using formula (1), and transformed using Eq. (2) with the chosen intrinsic parameters. Two-dimensional Gaussian noise with zero mean and standard deviation $\sigma$ is added to each image point $\hat{\mathbf{x}}$. Fig. 4 depicts two simulated images of three randomly generated lines. In Fig. 4A the visible arc has an amplitude $\theta=70^{\circ}$ and is sampled by 20 points. The camera intrinsic parameters appear in the bottom left corner. In Fig. 4B the visible arc is $\theta=140^{\circ}$ and the number of sample points is $N=140$. In this case the camera is not skewless. As a final remark notice that the amplitude of the visible arc is measured in the great circle where plane $\boldsymbol{\Pi}$ intersects the sphere, and not in the conic curve where the line is projected. In general the visible angle of the paracatadioptric line image is much less than $\theta$.

\subsection{Calibration of skewless camera with known aspect ratio}

Consider a skewless parabolic camera with aspect ratio $1.21\left(s_{k}=0\right.$ and $\left.r_{c}=1.21\right)$. Both the skew and the aspect ratio are assumed to be known. We aim to determine the focal length $\left(f_{c}=245\right)$ and the image center $\left(\left(c_{x}, c_{y}\right)=(330,238)\right)$ using the image of three lines $(K=3)$. The line projections are estimated by minimizing the sum of the square of the algebraic distances $\dot{\epsilon}$. The objective function is provided in Eq. (19) and the minimization problem has a closed form solution. The system is calibrated using the algorithm presented in Table 1 after estimating the conic curves where the lines are projected. The data points are synthetically generated using the simulation scheme explained above. The image of Fig. 4A is an example of a test image. The estimated calibration parameters are compared with the ground truth and the RMS error is computed over 100 runs of each experiment.

Fig. 5 shows the results for different choices of $\theta$ (amplitude of the visible arc) and $N$ (number of sample points).
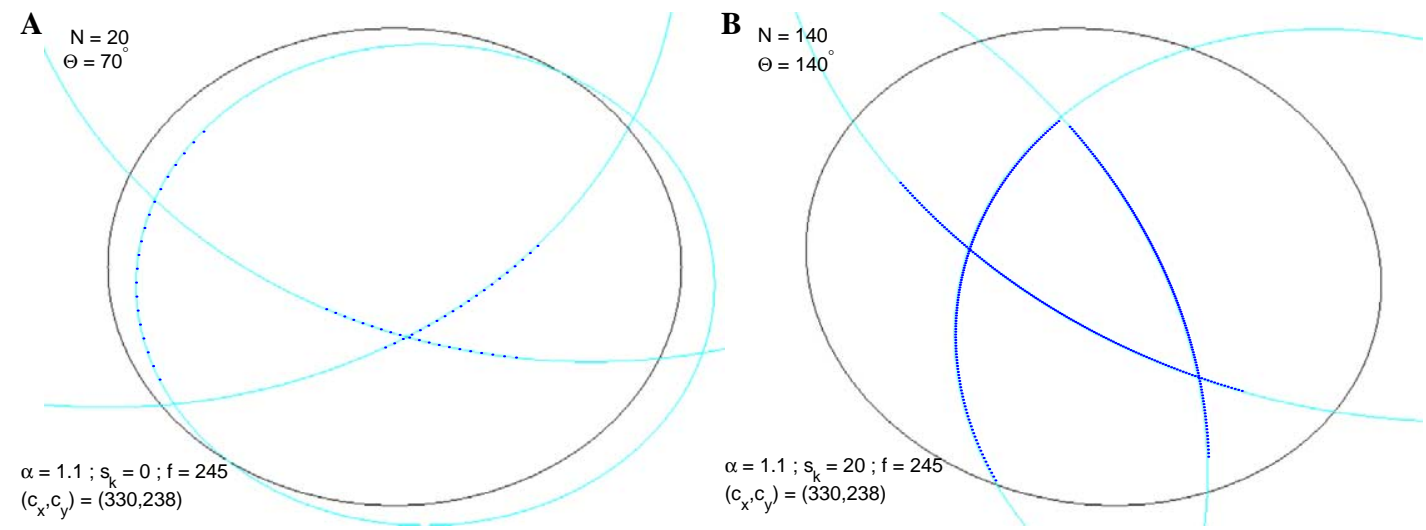

Fig. 4. Synthetic generation of $480 \times 640$ test images. (A) Test image (Section 5.2). (B) Test image (Section 5.3). 

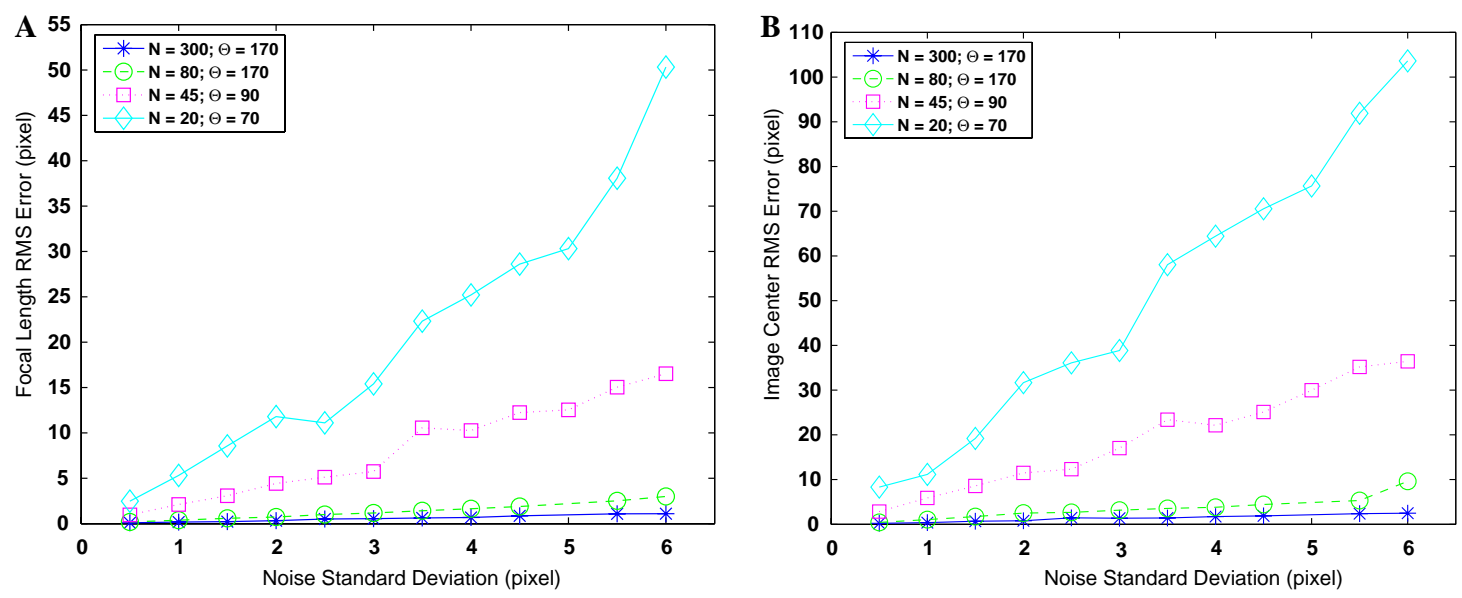

Fig. 5. Calibration of a skewless paracatadioptric camera with known aspect ratio using the image of three lines. The set of line projections is estimated using the closed form algorithm of section 3.3.2. The graphics show the root mean square (RMS) error for the focal length and image center. (A) Focal length. (B) Image center.

For each choice of $\theta$ and $N$ the standard deviation of the additive Gaussian noise varies between 0.5 and 6 pixels by increments of 0.5 pixels. For $\theta=170^{\circ}$ the algorithm presents an excellent performance. The decrease in the number of sample points from 300 to 80 only slightly affects the robustness to noise. Since we are only using three lines, the decrease in the amplitude of the visible arc $\theta$ and in the number of points $N$ has a strong impact on the performance. Even so the calibration using arcs of $90^{\circ}$ is still practicable. The situation of $\theta=70^{\circ}$ and $N=20$, shown in Fig. 4A, is very extreme and leads to a bad estimation of the intrinsic parameters.

An alternative calibration approach is presented in [16]. The authors evaluate the performance of their algorithm using similar simulation conditions. A direct comparison can be made between the results presented in here and the ones presented in [16]. In general terms they estimate the conic curves by exploiting the fact that the image center must lie in the line going through the intersection points of any two line images. As discussed in Section 3, this condition is necessary, but not sufficient, for a set of conic curves to be the paracatadioptric projection of lines. Since the search space is not fully constrained, they need much more than three lines to calibrate the sensor. The results presented in Fig. 5 are obtained using the minimum theoretical number of lines for calibration $[13,16]$. Even so, and as far as we are able to judge from the results presented in [16], the performance of our approach seems to be significantly better.

\subsection{Calibration of general paracatadioptric systems}

In this section, it is assumed that nothing is known about the calibration parameters. We aim to determine the aspect ratio, skew, focal length and image center using the paracatadioptric image of a set of $K$ lines. The test images are generated using the simulation scheme explained in Section 5.1. Fig. 4B shows an example of a test image with the assumed camera intrinsic parameters at the bottom left corner. The set of line projections is estimated by minimizing the function $\epsilon_{g}$ provided in Eq. (17). As discussed, the minimization is stated as a non-linear least squares problem, and the minimum is found using iterative gradient descent methods (typically Gauss-Newton or Lenvenberg-Marquardt [23,25]). The initial estimate for the iterative method is the closed form solution that minimizes function $\epsilon$ Eq. (16). The set of line projections is used to determine the calibration parameters following the steps enunciated in Table 1. The results are compared with the ground truth and the median error is computed over 100 runs.

In the first experiment, the system calibration is performed using the projection of three lines $(K=3)$. The application of gradient descent methods to minimize function $\epsilon_{g}$ can be problematic in many ways $[23,25]$. The choice of the initial estimate is crucial to assure a correct convergence. The iterative process must start from a point close enough to the global minimum to avoid possible local minima and saddle points. The objective function has this type of singularities when there is not enough information and/ or the search problem is not properly constrained. By insufficient information we mean a small number of lines, data points strongly corrupted with noise and visible arcs with small amplitude or not sampled enough. Fig. 6A shows the failure of convergence over the 100 runs for each experiment. The run fails when the absolute conic $\hat{\boldsymbol{\Omega}}_{\infty}$, determined following the steps in Table 1 , is not positive definite and the Cholesky decomposition is not possible. This can only happen when the set of paracatadioptric line images is far from being correctly estimated. As expected, the convergence is strongly affected by the noise. Moreover, the decrease in the number of sample points also causes an increase in the number of failures. Remark that we are using the minimum number of lines required to calibrate a paracatadioptric system. Therefore, it is natural that often the minimization process does not converge correctly. Fig. 6B shows the median error in the estimation 

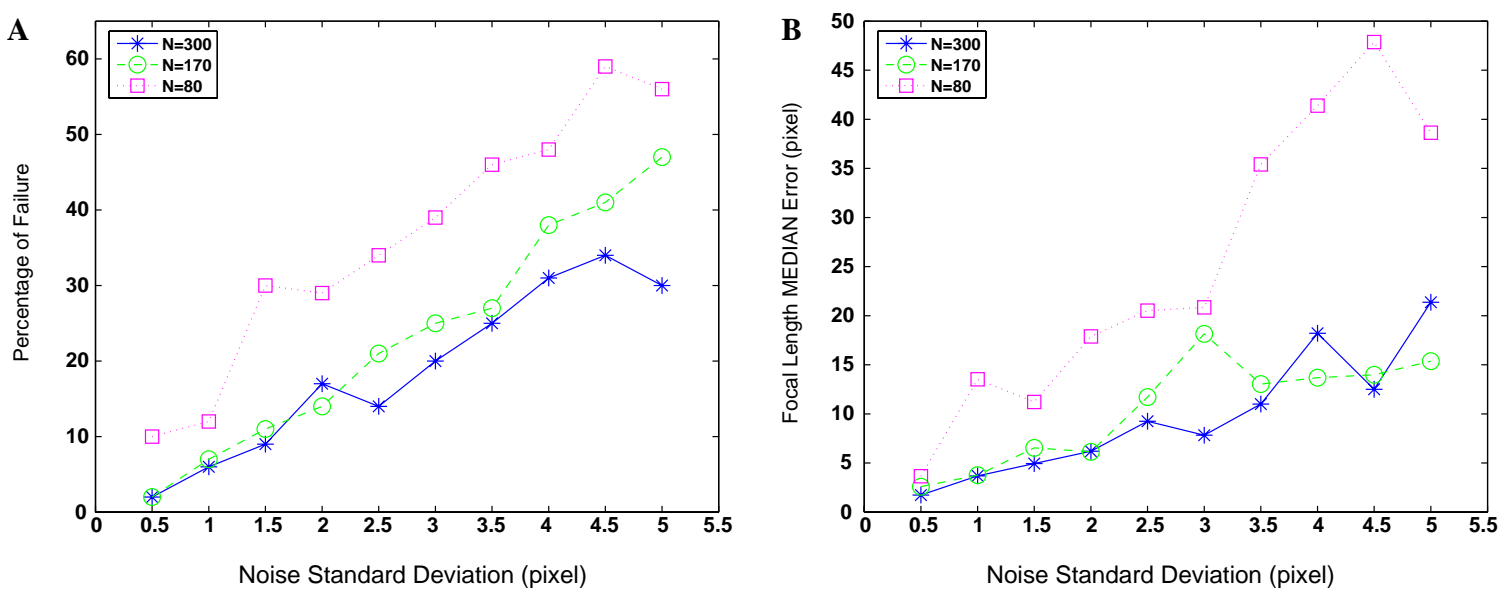

Fig. 6. Calibration using the projection of three lines $(K=3)$. The visible arc $\theta$ has $170^{\circ}$ of amplitude and the number of sample points $N$ is variable $(N=300,170,80)$. For each choice of $\theta$ and $N$ the standard deviation of the additive Gaussian noise varies between 0.5 and 6 pixels by increments of 0.5 pixels. (A) Failure rate in the convergence of the iterative gradient descent method. (B) Median error in the calibration of the focal length.

of the focal length with the performance decreasing with the number $N$ of sample points. The results are not very impressive because we are using only three line images.

The experiment of Fig. 7 compares the performance of the calibration algorithm when using the projection of 3 , 5,7 , and 9 lines. The increase of the number of lines dramatically improves the robustness of the calibration.
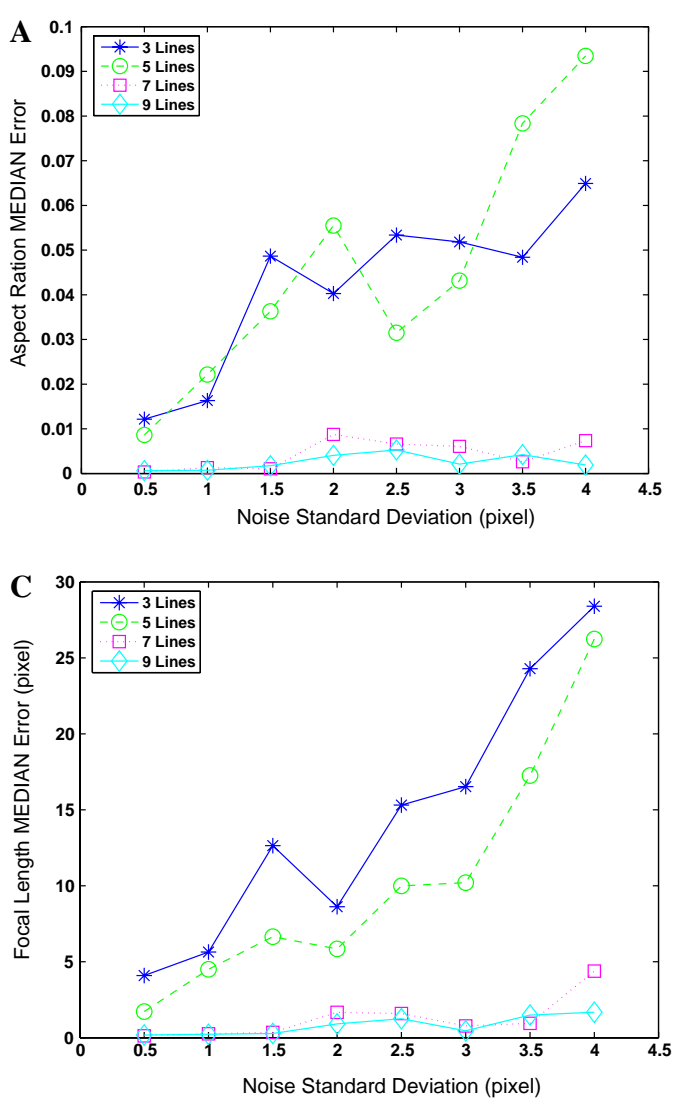

\subsection{Estimating paracatadioptric line projections in calibrated images}

The present section evaluates the performance of the fitting algorithm (CATPARB) proposed in Section 4. The synthetic data is generated using the simulation scheme explained in Section 5.1. Fig. 8 shows the estimation of a
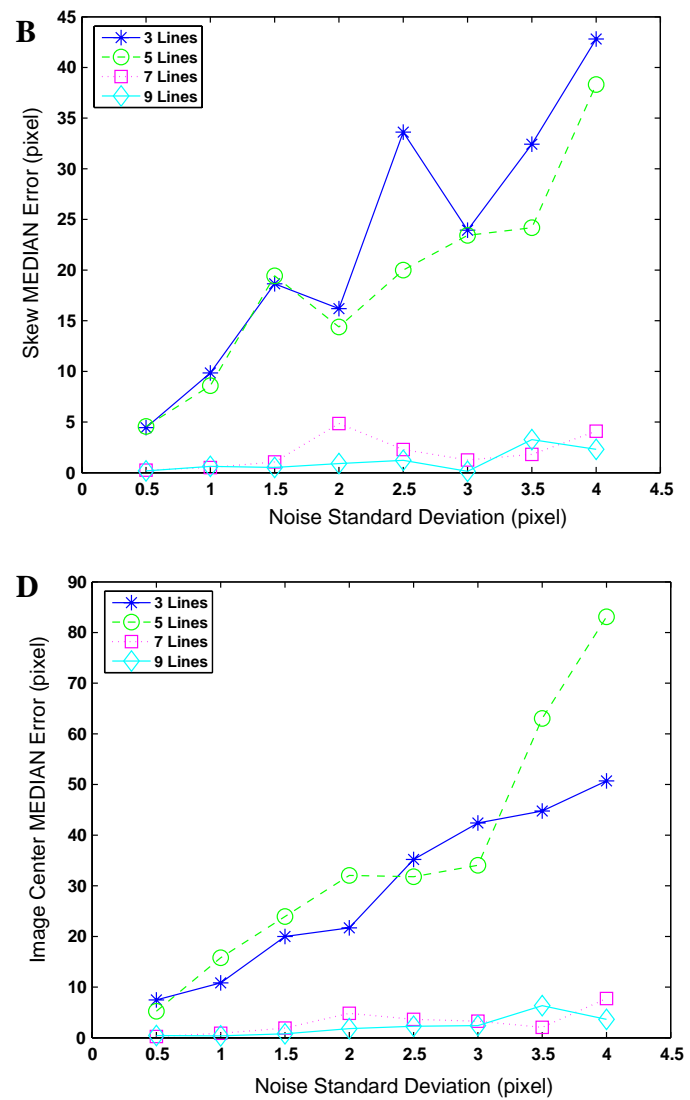

Fig. 7. Calibration using the projection of $K=3,5,7$, and 9 lines. The amplitude of the visible arc is $\theta=140^{\circ}$ and the number of sample points is $N=140$. The standard deviation of the additive Gaussian noise varies between 0.5 and 6 pixels. The graphics show the median error for the different calibration parameters. (A) Aspect ratio. (B) Camera skew. (C) Focal length. (D) Image center. 


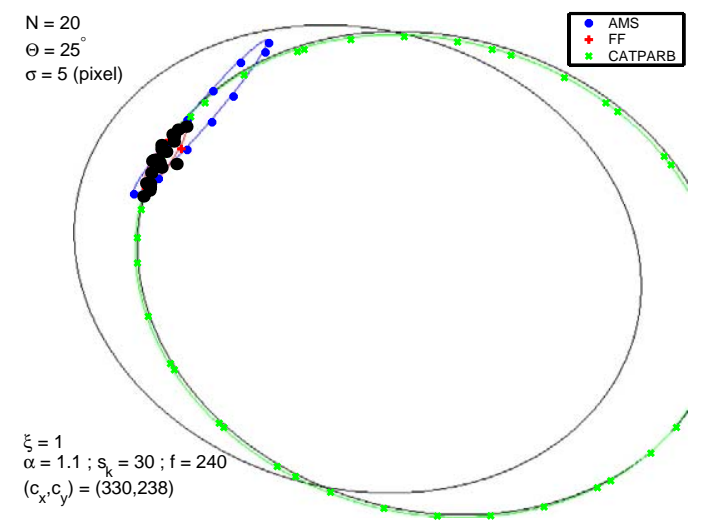

Fig. 8. Estimation of the conic curve corresponding to a line projection in a calibrated paracatadioptric image. The figure compares the estimation results using AMS, FF, and CATPARB. The amplitude of the visible arc is $\theta=25^{\circ}$, the number of sample points is $N=20$ and the standard deviation of the additive noise is $\sigma=5$ pixels.

paracatadioptric line projection using AMS, FF (Section 2.2), and CATPARB. The standard conic fitting methods perform poorly, but the CATPARB algorithm is able to correctly recover the conic curve. To certain extent the comparison of Fig. 8 is not entirely fair. While AMS and $\mathrm{FF}$ are generic methods to fit a conic curve to data points, CATPARB uses information about the sensor geometry and calibration to perform the estimation. The CATPARB algorithm is a specific method for the estimation of paracatadioptric line projections that requires the calibration matrix $\mathbf{H}_{\mathbf{c}}$ to be known.

If the system calibration is known, then the line projection can be easily determined by performing the perspective rectification of the data points. Consider the image points $\hat{\mathbf{x}}_{\mathbf{i}}$, lying in the paracatadioptric line projection $\hat{\mathbf{\Omega}}$, the calibration matrix $\mathbf{H}_{\mathbf{c}}$ (Eq. (5)) and the inverse of function $\hbar$ (Eq. (1)). Since $\overline{\mathbf{x}}_{\mathbf{i}}=\mathbf{H}_{\mathbf{c}}^{-1} \hat{\mathbf{x}}_{\mathbf{i}}$ and $\mathbf{x}_{\mathbf{i}}=\hbar^{-1} \overline{\mathbf{x}}_{\mathbf{i}}$, then the formula to compute the rectified data points is

$\mathbf{x}_{\mathbf{i}}=\hbar^{-1}\left(\mathbf{H}_{\mathbf{c}}^{-1} \hat{\mathbf{x}}_{\mathbf{i}}\right) \quad i=1, \ldots, N$

with

$\hbar^{-1}(\overline{\mathbf{x}})=\left(2 \bar{x} \bar{z}, 2 \bar{y} \bar{z}, \bar{z}^{2}-\bar{x}^{2}-\bar{y}^{2}\right)^{t}$.

The line projected into conic $\hat{\mathbf{\Omega}}$ lies in a plane $\boldsymbol{\Pi}$ with normal $\mathbf{n}=\left(n_{x}, n_{y}, n_{z}\right)^{t}$ (Fig. 1). The rectified points $\mathbf{x}_{\mathbf{i}}$, with $i=1, \ldots, N$, are projective rays in the plane $\Pi$ that satisfy $\mathbf{n}^{t} \mathbf{x}_{\mathbf{i}}=0$. Therefore, the normal $\mathbf{n}$ can be estimated from the set of rectified image points $\mathbf{x}_{\mathbf{i}}$ using normal least squares. The solution is the eigenvector of the matrix $\mathbf{C}$ (Eq. (26)) corresponding to the smallest eigenvalue. The conic locus $\hat{\boldsymbol{\Omega}}$ in the image plane is computed from $\mathbf{n}=\left(n_{x}, n_{y}, n_{z}\right)^{t}$ and $\mathbf{H}_{\mathbf{c}}$ using the relations established in Eqs. (3) and (4). Henceforth, we will refer this approach as the direct line estimation (DLE) method.

$\mathbf{C}=\left[\begin{array}{lll}x_{1} & y_{1} & 1 \\ x_{2} & y_{2} & 1 \\ \vdots & \vdots & \vdots \\ x_{N} & y_{N} & 1\end{array}\right]$.
Figs. 9 and 10 compare the performances of the AMS, FF, DLE, and CATPARB algorithms. The DLE method performs much better than the standard conic fitting techniques (AMS and FF). This is explained by the fact that the DLE uses not only the data points, but also implicit information about the sensor and its calibration. However, the performance of the DLE is clearly worse than the performance of the CATPARB method. As explained in Section 2.2, it is reasonable to assume that the noise in the image points $\hat{\mathbf{x}}_{\mathbf{i}}=\left(\hat{x}_{i}, \hat{y}_{i}\right)^{t}$ is Gaussian, two-dimensional and with zero mean. It is also reasonable to assume that

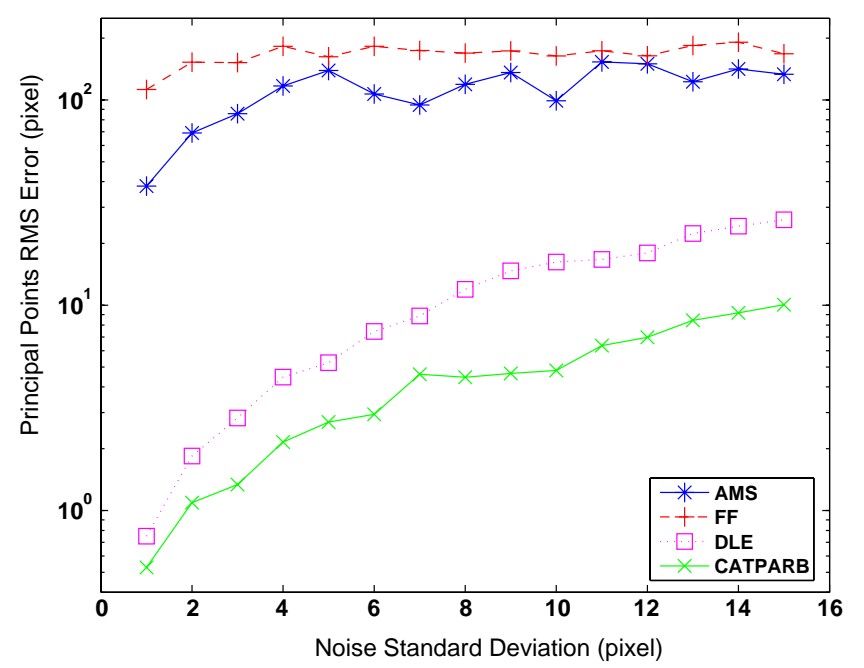

Fig. 9. Comparing the performance of AMS, FF, DLE, and CATPARB algorithms in estimating the conic locus where a line is projected. The data points are synthetically generated using the simulation scheme of Section 5.1. The visible arc has amplitude $\theta=80^{\circ}$ and is uniformly sampled by $N=40$ points. Each algorithm fits a conic to the synthetic data points. The estimated conic is compared with the ground truth and the RMS error in the principal points is computed over 100 runs.

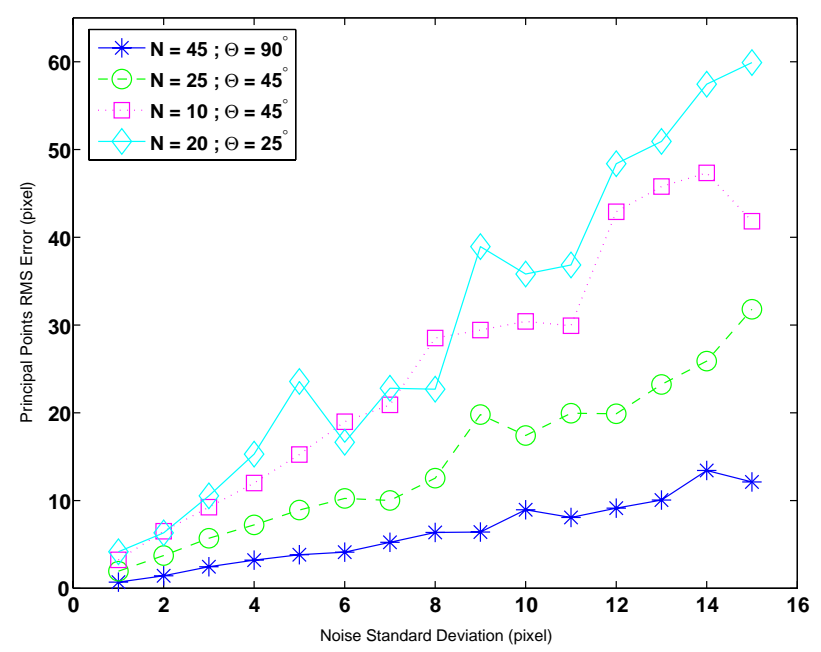

Fig. 10. Characterization of the performance of the CATPARB algorithm for different amplitudes of the visible $\operatorname{arc}(\theta)$ and number of sample points $(N)$. As expected the performance is worse when the number of samples and/or the amplitude of the visible arc decrease. The results provide a general idea of the robustness of the CATPARB algorithm. 
the error is equal in both directions and uncorrelated. Therefore, the noise covariance matrix is $\sigma^{2} \mathbf{I}$, where $\sigma$ is a scalar and $\mathbf{I}$ the $2 \times 2$ identity matrix. Consider the rectified point $\mathbf{x}_{\mathbf{i}}$ and the line $\mathbf{n}$, both lying in the conventional perspective plane. The algebraic distance between the point and the line is $\phi_{i}=\mathbf{n}^{t} \mathbf{x}_{\mathbf{i}}$. Replacing $\mathbf{x}_{\mathbf{i}}$ by the result of Eq. (25) and propagating the variance of the image point $\hat{\mathbf{x}}_{\mathbf{i}}$ follows that the noise variance in the algebraic distance is

$\sigma_{i}^{2}=4 \frac{\left(n_{x}^{2}+n_{y}^{2}\right)\left(\hat{x}_{i}^{2}+\hat{y}_{i}^{2}\right)^{2}+8 n_{x} n_{y} \hat{x}_{i} \hat{y}_{i}+2\left(n_{x}^{2}-n_{y}^{2}\right)\left(\hat{x}_{i}^{2}-\hat{y}_{i}^{2}\right)}{\left(1-\hat{x}_{i}^{2}-\hat{y}_{i}^{2}\right)^{4}} \sigma^{2}$

The least square estimator computes the line $\mathbf{n}$ that minimizes the sum of the squares of the algebraic distances $\phi_{i}$ $(i=1, \ldots, N)$. The estimation is optimal when the algebraic distances $\phi_{i}$ have the same noise variance and are statistically independent $[20,23]$. From Eq. (27) it follows that the variance $\sigma_{i}$ is a function of the coordinates of the original image point $\hat{\mathbf{x}}_{\mathbf{i}}$. Thus, the variance of the algebraic distances $\phi_{i}$ is not constant and the line estimation using least squares is statistically biased [24]. The effects of the statistical bias are much stronger in the DLE method than in the CATPARB algorithm, which explains the poorer performance of the former (Fig. 9).

\section{Experimental results using real images}

In this section we apply the calibration method proposed in Section 3 to determine the parameters of a real paracatadioptric sensor. Five images were taken using a paracatadioptric camera. Fig. 11 shows one of those images where a set of line projections is clearly visible. For each image we used an edge detector and selected points belonging to six different lines. Each one of the five images was independently calibrated using 4, 5, and 6 lines. The results are summarized in Table 2.

In this case, the calibration parameters are all unknown. The estimation of the line projections is performed by finding the solution that minimizes function $\epsilon_{g}$ of Eq. (17). The initial estimation for the iterative process is obtained using
Table 2

Calibration results using the projection of 4,5 , and 6 lines

\begin{tabular}{llrrrr}
\hline & $\mathbf{r}_{\mathbf{c}}$ & \multicolumn{1}{c}{$\mathbf{f}_{\mathbf{c}}$} & $\mathbf{s}_{\mathbf{k}}$ & \multicolumn{1}{c}{$\mathbf{c}_{\mathbf{x}}$} & \multicolumn{1}{c}{$\mathbf{c}_{\mathbf{y}}$} \\
\hline 4 Lines & & & & & \\
Mean & 1.0001 & 699.37 & 1.46 & 1137.6 & 870.66 \\
STD & 0.0012 & 16.00 & 2.35 & 22.6 & 13.42 \\
5 Lines & & & & & \\
Mean & 0.9998 & 701.03 & 0.57 & 1143.6 & 874.36 \\
STD & 0.0019 & 13.57 & 1.41 & 11.0 & 8.29 \\
6 Lines & & & & & \\
Mean & 0.9996 & 701.81 & -1.95 & 1147.7 & 876.64 \\
STD & 0.0015 & 10.65 & 1.39 & 5.8 & 5.66 \\
\hline
\end{tabular}

For each situation we performed five independent calibrations using five different images. The table shows the mean and standard deviation for each calibration parameter.

the AMS algorithm which, among the standard conic fitting methods with closed form solution, is the one that better performs in the presence of occlusion (see Section 2.2). Fig. 11A shows the initial estimates for the paracatadioptric line projections. If the conic loci were accurately determined then all the lines going through the intersection points should meet in the image center $([16,13])$. Fig. 11B exhibits the result corresponding to the minimum of function $\epsilon_{g}$. The calibration results are summarized in Table 2 . Notice that the estimated values for the calibration parameters are more or less the same for the different $K$ (number of lines). The standard deviation acts as a measure of confidence. If the standard deviation takes high values then the results obtained for each image are very different and the achieved calibration is not trustful. As expected the standard deviation decreases when the number of lines increases. Henceforth, we will assume that the camera is calibrated.

The experiment of Fig. 12 uses the CATPARB algorithm to determine the paracatadioptric projection of lines. Remark that the estimation results implicitly depend on the calibration accuracy. Therefore, we are simultaneously testing the CATPARB algorithm and the proposed calibration method. Since a line image has two independent degrees of freedom (Section 4), we select by hand two points lying on the conic locus where a scene line is project-
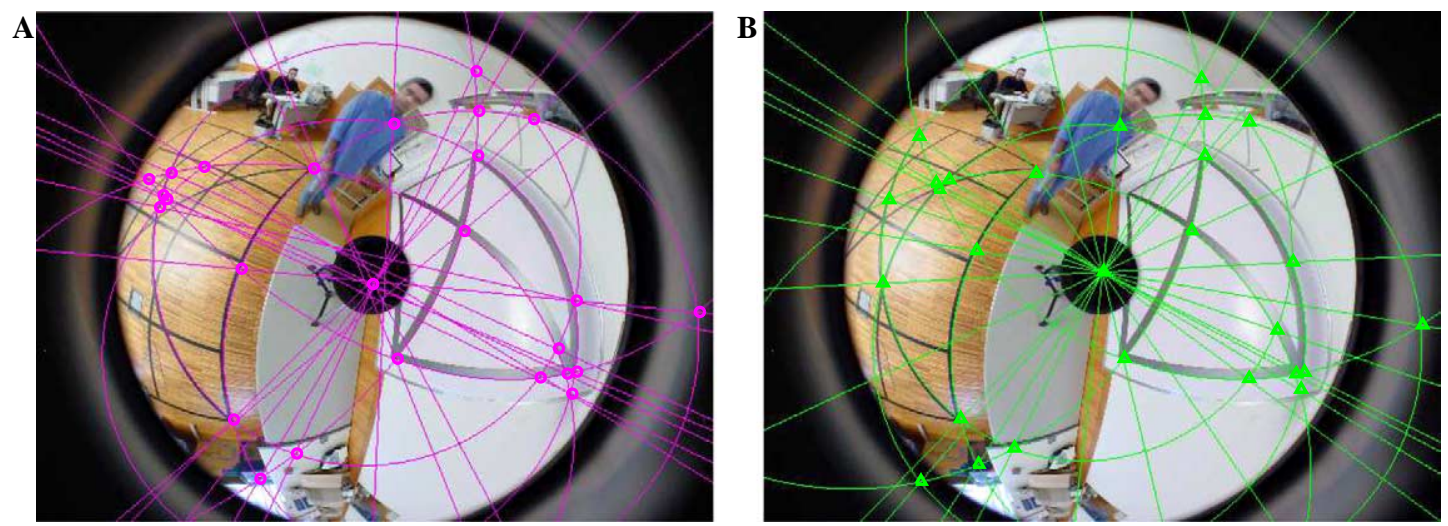

Fig. 11. Estimation of a set of line projections in an uncalibrated paracatadioptric image. The image resolution is $1704 \times 2272$ and the FOV is $180^{\circ}$. (A) Initial estimate (AMS). (B) Final estimate. 


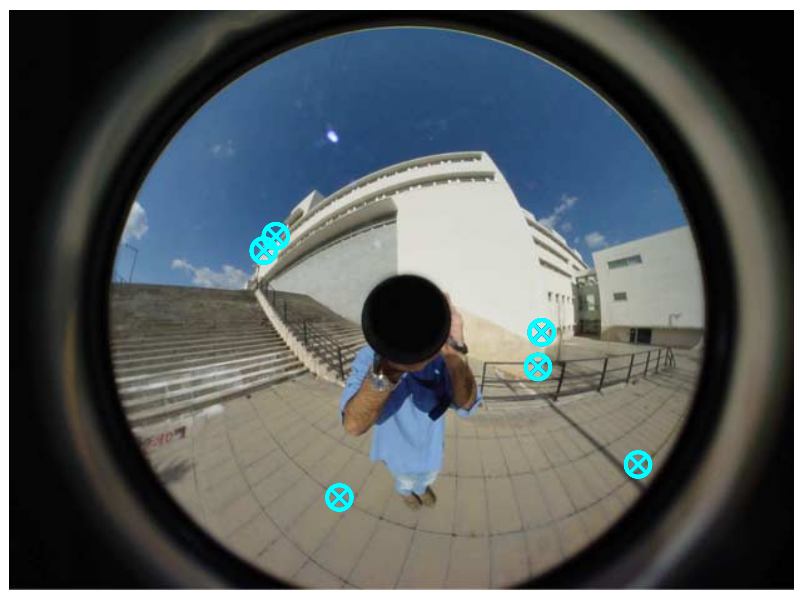

Fig. 12. Estimating the projection of lines in a calibrated paracatadioptric image using two points selected by hand.

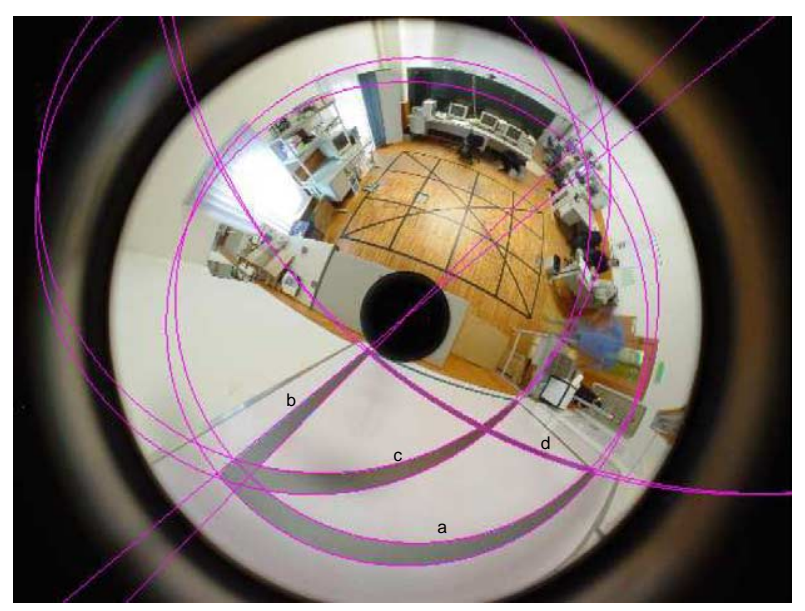

Fig. 13. Estimating the angles between pairs of parallel lines from a paracatadioptric image of those lines.

ed. Fig. 12 shows the selected points and the corresponding projected line estimated using CATPARB.

Fig. 13 is the paracatadioptric image of four pairs of parallel lines denote by $a, b, c$, and $d$. The polar of the image center with respect to the conic locus where each line is mapped is the horizon of the corresponding plane $\Pi$ containing the original 3D line and the effective viewpoint (see Fig. 1) $[13,10]$. Moreover, if two imaged lines are parallel then the intersection of the corresponding horizons is the vanishing point of their common direction. The eight line images, corresponding to the four pairs of parallel lines, are estimated using the CATPARB algorithm (see Fig. 13). The vanishing point of each pair is determined in a straightforward manner using the results of $[13,10]$. Since the calibration matrix $\mathbf{H}_{\mathbf{c}}$ is known, then the image of the absolute conic can be computed making $\hat{\boldsymbol{\Omega}}_{\infty}=\mathbf{H}_{\mathbf{c}}^{-t} \mathbf{H}_{\mathbf{c}}^{-1}$. The estimation of the angles between the pairs of parallel lines from the vanishing points and the absolute conic is trivial $[27,19]$. Table 3 shows the errors in estimating these angles.

There is an alternative approach to estimate the angles between the pairs of parallel lines. We can perform the perspective rectification of the image points, estimate the lines using normal linear least squares and compute the angles using standard projective relations. The estimation errors are exhibited in the second line of Table 3. As expected, estimating the lines directly in the paracatadioptric plane presents better results. We may conclude that the bias introduced by the perspective rectification has a strong impact on the performance of the DLE method.

\section{Conclusions}

This article presents an effective way to calibrate a paracatadioptric camera using the image of three or more lines in general position. It is shown that the accurate estimation of the conic curves where the lines are projected is hard to accomplish due to partial occlusion. We propose a strategy to overcome this difficulty. The necessary and sufficient conditions that must be verified by a set of conic curves to be the image of a set of lines are derived. These conditions are used to constrain the search space and accurately estimate the set of conic curves required to calibrate the paracatadioptric sensor. If the camera is skewless and the aspect ratio is known then the conic fitting problem is solved naturally by an eigensystem. Otherwise the estimation is performed using non-linear optimization techniques. Experimental results show that the proposed calibration method performs much better than the ones appearing in the literature [16-18].

The second contribution is the CATPARB algorithm to estimate the projection of a line in a calibrated paracatadioptric plane. It is proved that a conic curve, parameterized by a point in $\mathbf{P}^{5}$, is the paracatadioptric image of a line if and only if it lies in a hyperplane defined by the system parameters. Thus, there are three necessary and sufficient conditions which define a linear subspace in the space of all conic curves. The line image is estimated within this subspace by solving an eigensystem. The method is

Table 3

Recovering the angles between pairs of parallel lines

\begin{tabular}{lcccccrrr}
\hline & a-b & a-c & a-d & b-c & b-d & c-d & Mean & $S T D$ \\
\hline G. Truth & $90^{\circ}$ & $30^{\circ}$ & $30^{\circ}$ & $60^{\circ}$ & $60^{\circ}$ & $60^{\circ}$ & $3.87^{\circ}$ & $2.10^{\circ}$ \\
Error CAT & $0.85^{\circ}$ & $2.05^{\circ}$ & $1.82^{\circ}$ & $2.99^{\circ}$ & $1.04^{\circ}$ & $3.87^{\circ}$ \\
Error PER & $0.34^{\circ}$ & $6.64^{\circ}$ & $0.49^{\circ}$ & $2.40^{\circ}$ & $13.71^{\circ}$ & $16.01^{\circ}$ & $6.62^{\circ}$ \\
\hline
\end{tabular}

CAT denotes the results obtained by estimating the paracatadioptric projection of the lines. PER are the angles estimated after performing perspective rectification. 
accurate, robust and computationally efficient. Experimental results show that this approach performs much better than estimating the lines using perspective rectification as is often done in robotic applications [4]. The estimation after perspective rectification is statistically biased [24] which strongly affects the results.

\section{References}

[1] S. Baker, S. Nayar, A theory of catadioptric image formation, in: Proc. IEEE Internat. Conf. Computer Vision, Bombay, 1998, pp. 35 - 42.

[2] P. Sturm, A method for 3D reconstruction of piecewise planar objects from single panoramic images, in: Proc. IEEE Workshop on Omnidirectional Vision, Hilton Head Island, USA, 2000, pp. 119-126.

[3] P. Sturm, Mixing catadioptric and perspective cameras, in: Proc. IEEE Workshop on Omnidirectional Vision, Copenhagen, Denmark, 2002.

[4] J. Spletzer, A. Das, R. Fierro, C. Taylor, V. Kumar, J. Ostrowski, Cooperative localization and control of multi-robot manipulation, in Proc. IEEE Internat. Conf. on Intelligent Robots and Systems, Mauai, Hawaii, USA, 2001.

[5] T.E. Boult, R.J. Micheals, M. Eckmann, X. Gao, C. Power, S. Sablak, Omnidirectional video applications, in: Proc. 8th Internat. Symposium on Intelligent Robotic Systems, Reading, UK, 2000.

[6] A. Paulino, H. Araujo, Multiple robots in geometric formation: Control structure and sensing, in: Proc. 8th Internat. Symposium on Intelligent Robotic Systems, Reading, UK, 2000.

[7] J.P. Barreto, H. Araujo, A general framework for selecting world coordinate systems in perspective and catadioptric imaging application, Internat. J. Comput. Vis.

[8] J.P. Barreto, F. Martin, R. Horaud, Visual servoing/tracking using central catadioptric images, in: ISER2002-Proc. 8th Internat. Symposium on Experimental Robotics, Ischia, Italy, 2002.

[9] C. Geyer, K. Daniilidis, An unifying theory for central panoramic systems and practical implications, in: Proc. Eur. Conf. Comput. Vis., Dublin, 2000, pp. 445-461.

[10] J.P. Barreto, General Central Projection Systems: Modeling, Calibration and Visual Servoing, Ph.D. Thesis-University of Coimbra, 2003
[11] T. Svoboda, T. Pajdla, V. Hlavac, Epipolar geometry for panoramic cameras, in: Proc. Eur. Conf. Comput. Vis., Freiburg, Germany, 1998, pp. 218-332.

[12] C. Geyer, K. Daniilidis, Catadioptric projective geometry, Internat. J. Comput. Vis. 43 (2001) 223-243.

[13] J.P. Barreto, H. Araujo, Geometric properties of central catadioptric line images and their application for calibration, IEEE Trans. Pattern Anal. Mach. Intell. 27 (8) (2005) 1327-1333.

[14] Z. Zhang, Parameter estimation techniques: a tutorial with application to conic fitting, in: INRIA Raport de Recherche n 2676, 1995.

[15] A. Fitzgibbon, R. Fisher, A buyer's guide to conic fitting, in: Proc. of British Machine Vision Conference, Birmingham, England, 1995.

[16] C. Geyer, K. Daniilidis, Paracatadioptric camera calibration, IEEE Trans. Pattern Anal. Mach. Intell. 24 (4).

[17] S.B. Kang, Catadioptric self calibration, in: Proc. IEEE Internat. Conf. on Computer Vision and Pattern Recognition, USA, 2000, pp. 201-207.

[18] Y. Yagi, S. Kawato, S. Tsuji, Real-time omnidirectional vision sensor (copis) for vision guided navigation, IEEE Trans. Robot. Automat. 10

[19] J.G. Semple, G.T. Kneebone, Algebraic Projective Geometry, Claredon Press, Oxford, 1998.

[20] A. Papoulis, Probability, Random Variables, and Stochastic Processes, McGraw-Hill, New york, 1965.

[21] G. Taubin, Estimation of planar curves, surfaces and non planar space curves defined by implicit equations, with applications to edge and range image segmentation, IEEE Trans. Pattern Anal. Mach. Intell. 13 (11).

[22] A. Fitzgibbon, M. Pilu, R. Fisher, Direct least square fitting of ellipses, IEEE Trans. Pattern Anal. Mach. Intell. 21 (5).

[23] W. Press, S. Teukolsky, W. Vetterling, B. Flannery, Numerical Recipes in $\mathrm{C}++$, second Edition., Cambridge University Press, Cambridge, 2002.

[24] K. Kanatani, Statistical bias of conic fitting and renormalization, IEEE Trans. Pattern Anal. Mach. Intell. 16 (3).

[25] D. Schmabel, Numerical Methods for Unconstrained Optimization and Non-Linear Equations, Classics in Applied Mathematics.

[26] G.H. Golub, C.F.V. Loan, Matrix Computations, Johns Hopkins University Press, Baltimore, MD, 1983.

[27] R. Hartley, A. Zisserman, Multiple View Geometry in Computer Vision, Cambridge University Press, Cambridge, 2000. 